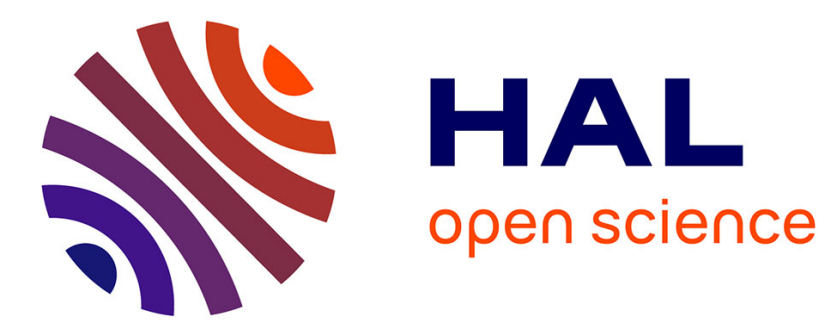

\title{
Estimators and confidence intervals for plant area density at voxel scale with T-LiDAR
}

François Pimont, Denis Allard, Maxime Soma, Jean-Luc Dupuy

\section{To cite this version:}

François Pimont, Denis Allard, Maxime Soma, Jean-Luc Dupuy. Estimators and confidence intervals for plant area density at voxel scale with T-LiDAR. Remote Sensing of Environment, 2018, 215, pp.343-370. 10.1016/j.rse.2018.06.024 . hal-02627515

\section{HAL Id: hal-02627515 https://hal.inrae.fr/hal-02627515}

Submitted on 6 Jan 2022

HAL is a multi-disciplinary open access archive for the deposit and dissemination of scientific research documents, whether they are published or not. The documents may come from teaching and research institutions in France or abroad, or from public or private research centers.
L'archive ouverte pluridisciplinaire HAL, est destinée au dépôt et à la diffusion de documents scientifiques de niveau recherche, publiés ou non, émanant des établissements d'enseignement et de recherche français ou étrangers, des laboratoires publics ou privés.

\section{(ㅇ)(1) $\$$}

Distributed under a Creative Commons Attribution - NonCommercial - NoDerivatives| 4.0 
1 Estimators and confidence intervals for plant area density at voxel scale with T-LiDAR

2

$5 \quad{ }^{1}$ URFM, INRA, 84914, Avignon, France

$6 \quad{ }^{2}$ BioSP, INRA, 84914, Avignon, France

$7 \quad{ }^{*}$ Corresponding author. francois.pimont@inra.fr

\section{Abstract:}

10 Terrestrial LiDAR becomes more and more popular to estimate leaf and plant area density.

11 Voxel-based approaches account for this vegetation heterogeneity and significant work has 12 been done in this recent research field, but no general theoretical analysis is available.

13 Although estimators have been proposed and several causes of biases have been identified,

14 their consistency and efficiency have not been evaluated. Also, confidence intervals are 15 almost never provided.

16 In the present paper, we solve the transmittance equation and use the Maximum Likelihood

17 Estimation (MLE), to derive unbiased estimators and confidence intervals for the attenuation coefficient, which is proportional to leaf area density. The new estimators and confidence

19 intervals are defined at voxel scale, and account for the number of beams crossing the voxel, 20 the inequality of path lengths in voxel, the size of vegetation elements, as well as for the 21 variability of element positions between vegetation samples. They are completed by 22 numerous numerical simulations for the evaluation of estimator consistency and efficiency, as 23 well as the assessment of the coverage probabilities of confidence intervals.

24 - Although commonly used when the beam number is low, the usual estimators are strongly 25 biased and the $95 \%$ confidence intervals can be $\approx \pm 100 \%$ of the estimate. 
- Our unbiased estimators are consistent in a wider range of validity than the usual ones, especially for the unbiased MLE, which is consistent when the beam number is as low as 5 . The unbiased MLE is efficient, meaning it reaches the lowest residual errors that can be expected (for an unbiased estimator). Also the unbiased MLE does not require any bias correction when path lengths are unequal.

- When elements are small (or voxel is large), $10^{3}$ beams entering the voxel leads to some confidence intervals $\approx \pm 10 \%$, but when elements are larger (or voxel smaller), it can remain wider than $\pm 50 \%$, even for a large beam number. This is explained by the variability of element positions between vegetation samples. Such a result shows that a significant part of residual error can be explained by random effects.

- Confidence intervals are much smaller ( \pm 5 to $10 \%$ ) when LAD estimates are averaged over several small voxels, typically within a horizontal layer or in the crown of individual plants. In this context, our unbiased estimators show a reduction of $50 \%$ of the radius of confidence intervals, in comparison to usual estimators.

Our study provides some new ready-to-use estimators and confidence intervals for attenuation coefficients, which are consistent and efficient within a fairly large range of parameter values. The consistency is achieved for a low beam number, which is promising for application to airborne LiDAR data. They entail to raise the level of understanding and confidence on LAD estimation. Among other applications, their usage should help determine the most suitable voxel size, for given vegetation types and scanning density, whereas existing guidelines are highly variable among studies, probably because of differences in vegetation, scanning design and estimators. 
Highlights:

- Voxel-based estimations of LAD/PAD may lack of consistency and efficiency

- We propose new estimators based on theoretical derivation and numerical simulations

- Estimators for confidence intervals are also provided

- New estimators should help determine the most appropriate voxel resolution

\section{$\underline{\text { 1. Introduction }}$}

The amount and spatial distribution of foliage in a tree canopy have a fundamental function in ecosystems by affecting energy and mass fluxes through photosynthesis and transpiration. Moreover, canopy structure may reveal plant adaptation strategies to their physical or biotic environment (Norman and Campbell, 1989). Canopy foliage has other important ecological functions since it constitutes the crown fuels involved in high intensity forest fires (Keane, 1995) and its spatial structure may determine the habitat quality for animal species (Vierling et al., 2008). Terrestrial LiDAR (Light Detection And Ranging), referred to hereinafter as TLS (Terrestrial LiDAR System) recently emerged as a promising tool to estimate leaf/plant area density (LAD/PAD) distribution for individual plants and forest plots. Although similar traversal algorithms have recently been used with high resolution airborne data, acquisitions still suffer from substantial occlusion. This occlusion could be reduced with large flight strip overlaps (Kükenbrik et al., 2017), which would lead to a promising application of methods initially developed for TLS. Two classes of methods are commonly applied to derive LAD distributions with TLS. First, the leaf area density profile can be measured through a gap fraction approach (Jupp et al., 2009; Zhao et al., 2011). Rigorous statistical analysis using maximum likelihood estimator (MLE) has been applied to the gap fraction equation inversion, leading to robust estimates of LAD and leaf angulation profiles (Zhao et al., 2015). 
Unfortunately, the gap fraction approach does not explicitly account for spatial correlation in vegetation distribution (Zhao et al., 2015), whereas spatial correlation in heterogeneous media are known to modify transmission laws and free path distribution (Davis and Marshak, 2004; Pimont et al., 2009; Larsen and Clark, 2014). A clumping factor is thus required (Chen and Cihlar, 1995; Zhao et al., 2011). Stochastic geometry entails to explicitly account for such clumping, but only to determine the leaf area index, LAI (Allard et al., 2013), which is the integral of the LAD over the vertical. The second class of methods is voxel-based and explicitly account for clumping at scales larger than voxel size. They entail to assess not only the vegetation vertical profile, but the full 3D distribution of area or mass density. Several approaches have been developed: the voxel-based profiling (Hosoi and Omasa, 2006 \& 2007; Bailey and Mahafee, 2017a), the relative density index (Durrieu et al., 2008; Pimont et al., 2015), the modified contact frequency (Béland et al., 2011) and the Beer-Lambert approach (Béland et al., 2014b; Grau et al., 2017; Bailey and Mahafee, 2017a). These theoretical indices can be readily applied or combined with field measurements through a calibration phase (e.g., in Pimont et al., 2015).

The application of physical principles such as turbid media and contact frequency to voxelized-TLS data raises several problems that folds in two categories. The first one deals with departure from ideal measurements due to TLS "flaws". An idealized TLS would send an infinite number of infinitely thin beams on any voxel. The actual diameter of the beam (on the order of a few mm) is responsible for partial hits (Hebert and Koktov, 1992; Béland et al., 2011; Grau et al., 2017). There is also uncertainty regarding beam intensity, due to the noise instrument gain, that affects the detection (Grau et al., 2017). Béland et al., (2011) proposed an approach that accounts for partial hits and intensity through a calibration of intensity and view factors. Another aspect that has received little attention until now is the number of beams entering the voxel (sampling size). The beam number depends on the distance to 
101 scanner, the direction and scanner resolution, as well as the interaction with vegetation which

102 limits the number of beams reaching a given background voxel (occlusion). A basic rule of

103 the thumb is to only consider measurements with beam number larger than 10 (Béland et al.,

104 2014a) or to compute indices in large voxels, which leads to a fairly large beam number in 105 most cases (Bailey and Mahafee, 2017a).

106 The second cause of departure from ideal measurement is vegetation "flaws". An idealized 107 vegetation would be made of leaf only, assumed to be infinitely small elements with random 108 distribution of position and orientation. The actual orientation and size of elements alter 109 transmission laws (Larsen and Clark, 2014) and can be accounted for as in Béland et al.

110 (2011), where leaf orientation is separately measured and the interaction between a single leaf 111 and a beam is modelled. Element and branch orientations have been reported to be of 112 secondary importance in comparison to other sources of errors (Grau et al., 2017; Seielstadt et 113 al., 2011; Pimont et al., 2015). However, the assumption of spherical leaf inclination is not 114 valid in many cases (Pisek et al., 2013), which suggests that the assessment of the proper 115 angle distribution is likely to reduce errors. A recent method based on triangulation entails to 116 estimate the orientation factor with a TLS, provided that leaves are large enough to be 117 individually sampled by several beams (Bailey and Mahafee, 2017b). Regarding element size,

118 Grau et al. (2017) reports little effects when elements are much smaller than grid size. This 119 effect has been demonstrated to vary with voxel size (Béland et al., 2014b). Finally, several 120 methods based on return intensity have been proposed to separate leaf from wood returns and 121 to account for it (Béland et al., 2011, 2014a), even though such a method can not be applied to

122 all TLS (Pimont et al., 2015). Despite these known issues, a detailed analysis of the 123 consistency is still missing.

124 The determination of confidence intervals on LAD estimates has received little attention until 125 now. If such estimators are known in the context of gap fraction approaches (Zhao et al., 
126 2015), confidence interval for voxel-based approaches are seldom proposed (with the 127 exception of Pimont et al., 2015). Most error evaluations are based on simple comparison to 128 experimental data, in which various sources of bias and dispersion may interact. This might 129 explain why there is no consensus about the selection of voxel size among studies (e.g., 130 Béland et al., 2011, 2014a; Bailey and Mahafee, 2017a; Grau et al., 2017). More generally, a 131 rigorous statistical analysis of estimators such as in Zhao et al. (2015) is still missing for voxel-based approaches.

133 In the present study, we focus on some of the vegetation "flaws". We set our approach in the

134 framework of random set theory, stochastic geometry and stereology (Stoyan et al., 1987;

135 Schneider and Weil, 2008). In stochastic geometry, random distributions of geometrical 136 objects such as points, segments and disks are analyzed and analytical expressions are derived 137 for geometrical characteristics such as mean volume and area, specific surface etc. Stereology 138 is concerned with the estimation of those quantities with limited probing, in particular in 139 lower dimensions, such as beams probing a canopy voxel. We develop generalized estimators 140 towards two different approaches: i) the resolution of the transmittance equation (also called 141 Beer-Lambert law), ii) the maximum likelihood. Our developments are theoretical and 142 validated through numerical simulations. They include bias corrections for the beam number, 143 the element size, as well as for the variability of element positions between vegetation 144 samples. Estimators for variance are also provided and can be used to compute confidence 145 intervals. In order to facilitate the reading of the manuscript, most of the mathematical 146 development are detailed in supplementary materials for reference and only the main 147 equations are presented in the manuscript. Numerical simulations are used to compare the 148 new estimators to usual ones (Beer-Lambert, Modified Contact Frequency), through the 149 analysis of their consistency (i.e. bias size) and of their efficiency at the scale of a single voxel 150 or a group of voxels (i.e. $95 \%$ error). The application of the new estimators and their 
151 confidence interval are then discussed, especially in the context of the determination of the 152 most appropriate voxel size.

153

154 2. Background regarding the estimation of PAD/LAD through the attenuation

155 coefficient

156 This section summarizes the existing knowledge regarding the estimation of attenuation coefficient in voxel-based approaches and defines a few notations.

158

\subsection{Beer-Lambert law formulation for TLS}

160 The transmittance $\mathrm{T}$ in small and randomly distributed vegetation elements with no scattering

161 follows an exponential attenuation along a path of length $\delta$, known as the Beer-Lambert law 162 (Nilson, 1971; Ross, 1981):

$$
\mathrm{T}=\mathrm{e}^{-\int_{0}^{\delta} \lambda(\mathrm{z}) \mathrm{dz}}
$$

163 with $\lambda$ the attenuation coefficient of the medium $\left(\mathrm{m}^{-1}\right)$

164 The plant area density $\left(\mathrm{PAD}, \mathrm{m}^{-1}\right)$ is related to the attenuation coefficient $\left(\lambda, \mathrm{m}^{-1}\right)$ :

$$
\mathrm{PAD}=\lambda / \mathrm{G}
$$

165 where $G$ is the plant projection function, which is frequently assumed to be equal to 0.5 .

166

167 The complementary to one of the transmittance is the absorbance, A. TLS can be used to

168 estimate the absorbance of a vegetation sample with the relative density index I (also denoted

169 RDI in text), defined for a volume of vegetation, further referred to as the voxel $V$ with

170 volume denoted $|V|$. The RDI is the ratio between the number of hits within the voxel $\left(\mathrm{N}_{\mathrm{i}}\right)$, to

171 the number of beams that reaches the voxel $(\mathrm{N})$ :

$$
1-\mathrm{T}(\lambda)=\mathrm{A}(\lambda) \approx \mathrm{I}=\frac{\mathrm{N}_{\mathrm{i}}}{\mathrm{N}}
$$


172 When beams are aligned with one cell face for cubic voxels or when the geometry of voxels is

173

174

175

$$
\mathrm{T} \approx \mathrm{e}^{-\lambda \bar{\delta}}
$$

176

where $\bar{\delta}$ is the mean path length within the voxel.

177 Taking the logarithm of the transmittance equation (Eq. 4) and combining with Eq. 3 leads to

178 the usual estimator of the attenuation coefficient:

$$
\hat{\lambda}=-\frac{\log (1-\mathrm{I})}{\bar{\delta}}
$$

179 This estimator (later referred to as the usual Beer-Lambert estimator) assumes that the

180 attenuation coefficient is constant in the voxel, that the vegetation elements are infinitely small and that path length variations are negligible. Unequal path lengths involve the variance of path length within voxels (Grau et al., 2017). An empirical correction that depends on voxel orientation is described in Béland et al. (2014b) for cubic voxels. Another approach is

184 to use the secant method to solve the exponentially-weighted transmittance equation, since 185 such an equation does not have an explicit solution (Bailey and Mahafee, 2017a).

$186 \hat{\lambda}$ is not defined when $\mathrm{I}=1$, i.e. when no beam travels beyond the voxel. This occurs with 187 probability $\left(1-\mathrm{e}^{-\lambda \delta}\right)^{\mathrm{N}}$ which is, for example, equal to 0.01 when $\lambda \delta=1$ and $\mathrm{N}=10$.

188 Although this probability is very low when $\mathrm{N}$ is high, such an event may happen quite often in 189 any large voxelized scene, especially when the vegetation is dense. These cases can simply be 190 ignored, as proposed in Béland et al. (2014a), considering these cases as "occluded", but it 191 will be shown later that it leads to biases and loss of efficiency. 
194 The contact frequency of vegetation elements CF is the number of contacts per unit length of

195 point quadrat (probe) (Warren Wilson, 1960):

$$
\mathrm{CF}=\frac{\sum_{\mathrm{j}=1}^{\mathrm{N}} \mathrm{C}_{\mathrm{j}}^{\mathrm{l}}}{\mathrm{N} \delta}
$$

196 where $C_{j}^{l}$ is the number of leaf contacts for the $j^{\text {th }}$ probing and $\delta$ the probe length, and 197 assuming that the probing number $\mathrm{N}$ is large. It is related to plant area density in a similar 198 manner as the attenuation coefficient:

$$
\mathrm{PAD} \approx \mathrm{CF} / \mathrm{G}
$$

With a TLS, the laser represents a virtual probe that is intercepted by vegetation. However, the contact number cannot exceed one and only a fraction of the volume is explored by the beam. A direct application of this method with TLS thus leads to an underestimation of the attenuation coefficient (Bailey and Mahafee, 2017a). This method is adapted in Béland et al.,

203 (2011) for TLS data, thank to the volume fraction concept and leads to:

$$
\tilde{\lambda}=\frac{\sum_{j=1}^{N} \mathbf{1}_{z_{j}<\delta_{j}}}{\sum_{j=1}^{N} z_{j}}=\frac{N_{i}}{N \bar{z}}=\frac{I}{\bar{z}}
$$

204 where $\mathrm{z}_{\mathrm{j}}$ is the length of the path actually explored (free path) by the $\mathrm{j}^{\text {th }}$ beam and $\mathbf{1}_{B}$ is the

205 indicator function of event $B\left(\mathbf{1}_{\mathrm{z}_{\mathrm{j}}<\delta_{\mathrm{j}}}=1\right.$ if the $\mathrm{j}^{\text {th }}$ beam hits vegetation inside the voxel and 0 206 otherwise).

207 This formulation assumes that the explored volume is statistically representative of the 208 unexplored volumes. However, it does not assume the equality of path lengths (contrary to 209 Beer-Lambert estimator).

\subsection{Accounting for the size of vegetation elements}

212 The finite size of elements (ie. the size of elements is larger than zero in the real world)

213 induces a bias in the above estimators when the element size is not negligible when compared 214 to voxel size (Béland et al., 2014a). Assuming that the beams are parallel, let $S_{1}$ and $S$ be 
215 respectively the cross sections of the element size and voxel volume, which will be assumed

216 constant for simplicity. The probability that a given beam crosses the voxel containing $\mathrm{p}$

217 elements is $\left(1-\frac{s_{1}}{s}\right)^{p}$, as shown in Campbell and Norman (1998, chapter 15).

218 The volume cross section is given by $\mathrm{S}=\frac{|\mathrm{V}|}{\delta}$, where $\delta$ is the path length. The contribution of 219 a single leaf to the attenuation coefficient of the voxel $\mathrm{V}$ is:

$$
\lambda_{1}=\frac{\mathrm{S}_{1}}{|\mathrm{~V}|}=\frac{\mathrm{S}_{1}}{\mathrm{~S}} \frac{1}{\delta}
$$

220 Since $\lambda=p \lambda_{1}$, the transmittance of the voxel is:

$$
\mathrm{T} \approx\left(1-\frac{\mathrm{S}_{1}}{\mathrm{~S}}\right)^{\mathrm{p}}=\left(1-\lambda_{1} \delta\right)^{\frac{\lambda}{\lambda_{1}}}
$$

221 When the element size is not neglected is, the Beer-Lambert estimator is modified as follow:

$$
\widehat{\lambda_{p}}=\frac{\lambda_{1} \log (1-\mathrm{I})}{\log \left(1-\lambda_{1} \delta\right)}
$$

222 As show with slightly different notations in Béland et al. (2014a), $\widehat{\lambda_{p}}=\frac{\widehat{\lambda}}{-\frac{1}{\lambda_{1} \delta} \log \left(1-\lambda_{1} \delta\right)}$, which

223 converges to $\hat{\lambda}$ when $\mathrm{R}=\frac{\mathrm{s}}{\mathrm{s}_{1}}=\frac{1}{\lambda_{1} \delta}$ is large, or equivalently, when $\lambda_{1} \delta$ tends to 0 .

In the discussion section of Béland et al. (2014a), the same correction factor is suggested to apply to the modified contact frequency, so that the modified contact frequency for finite size element would be:

$$
\widetilde{\lambda_{p}}=-\frac{\lambda_{1} \bar{\delta}}{\log \left(1-\lambda_{1} \bar{\delta}\right)} \frac{\mathrm{I}}{\overline{\mathrm{z}}}
$$

Depending whether elements are small needles or broad leaves, $\lambda_{1} \delta$ typically range between $230210^{-5} \delta^{-2}$ and $510^{-3} \delta^{-2}$ (See supplementary S1). This means that $\lambda_{1} \delta$ ranges between 2310.002 and 0.5 when the voxel size is about $10 \mathrm{~cm}$, and between 0.0002 and 0.05 when the 232 voxel size is $30 \mathrm{~cm} . \lambda_{1} \delta$ is smaller than 0.005 when the voxel size is on the order of $1 \mathrm{~m}$. 


\section{3. Mathematical formulation}

235

236 In this section, we develop the mathematical framework leading to unbiased estimators for

237 point, variance and confidence intervals of the attenuation coefficient. The proofs are given in

238 Supplementaries S2 and S3.

239

240

\subsection{Set-up and notations}

241 We assume a finite number of elements and we rely on the notations defined in 2.3. For a

242 given quantity $\mathrm{A}$, such as an estimator, a variance or a confidence interval radius, $\hat{A}$ denotes

243 the quantity as derived from the Beer-Lambert law, whereas $\tilde{A}$ is derived from the Maximum

244 Likelihood Estimator approach. As shown below, the MLE generalizes the Modified Contact

245 Frequency introduced in Béland et al. (2011), so that these symbols are consistent with

246 section 2 (Eq. 8). Furthermore, the use of upper case letters, such as $\Lambda$, refers to our new

247 estimators presented below, whereas lower case letters, such as $\lambda$, refers to the usual ones

248 presented in the background section.

249

250 We briefly present some stochastic geometry material and refer to the literature for a more

251 detailed exposition (Stoyan et al., 1987; Schneider and Weil, 2008). $p$ elementary objects

252 identical in shape and size are located at random within the voxel $V$. If one representative of

253 these objects is denoted $B$, the vegetation elements, denoted $X$, corresponds to the union of all

254 objects:

$$
X=\bigcup_{k=1}^{p} B\left(x_{k}\right)
$$

255 where $B\left(x_{k}\right)$ denotes the element $B$ shifted to the random location $x_{k} \in V$. 
257 One special case of interest is the Boolean model, for which the number of objects $p$ is 258 distributed according to a Poisson distribution (Stoyan et al., 1987). A remarkable feature of 259 Boolean models is that the intersection of a Boolean model by a random line is also a Boolean 260 model with intensity $\lambda=\mathrm{S}_{1} \frac{\mathrm{p}}{|\mathrm{V}|}$, where $\mathrm{S}_{1}$ is the cross-section of $B$ perpendicular to the line. As 261 a consequence, the lengths $Y$ of segments with no intersection with $X$, called free paths in the 262 present context, are distributed according to an exponential random variable with parameter $\lambda$ :

$$
\mathrm{P}(Y>y)=\mathrm{e}^{-\lambda y}, y>0
$$

263 When the object number is not assumed to be large, the distribution becomes:

$$
\mathrm{P}(Y>\mathrm{y})=\left(1-\lambda_{1} \mathrm{y}\right)^{\lambda / \lambda_{1}}
$$

264 with $\lambda_{1}=\frac{\mathrm{S}_{1}}{|\mathrm{~V}|}$.

265 Let $M$ be the segment corresponding to the intersection between a beam and the voxel $V$.

266 Depending on voxel shape and size, its length $\delta$ follows a distribution $\Delta$. The distance actually

267 traveled in voxel $\mathrm{Z}$ by a beam corresponds to:

$$
\mathrm{Z}=\min (Y, \Delta)
$$

268 The probability distribution of $\mathrm{Z}$ is derived from Eq. 15:

$$
\mathrm{f}_{Z}(\mathrm{z} ; \delta)=\left\{\begin{array}{c}
\lambda\left(1-\lambda_{1} \mathrm{z}\right)^{\lambda / \lambda_{1}-1} \text { when } \mathrm{z}<\delta \\
\left(1-\lambda_{1} \delta\right)^{\lambda / \lambda_{1}} \text { when } \mathrm{z}=\delta
\end{array}\right.
$$

269 In the $\mathrm{Z}$ distribution, the density of $\mathrm{Y}$ for $\mathrm{Y}>\delta$ is cumulated at $\mathrm{z}=\delta$ due to the "min" operator

270 in Eq. 16. Note that there are two components of randomness in this set-up: a random

271 realization of a Boolean model $X$, on the one hand (i.e. element positions for a vegetation

272 sample) and a random beam $M$ over the cross-section of the voxel $S$, on the other hand (i.e.

273 instrument sampling). 
275 Let us consider a given realization of the Boolean model $\mathrm{X}$ and $\mathrm{N}$ beams $\left\{m_{j}\right\}_{j \leq \mathrm{N}}$ distributed 276 over the voxel. We can define the RDI (Eq. 3) as the fraction of beams hitting the canopy

277 elements as:

$$
\mathrm{I}\left(X,\left\{m_{j}\right\}_{j \leq \mathrm{N}}\right)=\frac{\mathrm{N}_{\mathrm{i}}(X)}{\mathrm{N}}=\frac{\sum_{\mathrm{j}=1}^{\mathrm{N}} \mathbf{1}_{\mathrm{z}_{\mathrm{j}}\left(X, m_{j}\right)<\delta_{\mathrm{j}}\left(m_{j}\right)}}{\mathrm{N}}
$$

278 Let us now denote $\mathrm{I}_{\infty}(X)$ the asymptotic RDI, which is the expectation of $\mathrm{I}(X)$ with respect to

279 the instrument sampling (i.e. $\mathrm{N} \rightarrow \infty)$ :

$$
\mathrm{I}_{\infty}(\mathrm{X})=\mathrm{E}_{M}\left[\mathbf{1}_{\mathrm{Y}(X)<\Delta}\right]
$$

280

\subsection{Point, Variance and Confidence Interval of the relative density index (RDI)}

282 The RDI defined in Eq. (18) for $\mathrm{N}$ beams has the same expectation as the asymptotic RDI

283 over all configurations $\mathrm{X}$, since beams are drawn randomly across $S$.

284 This expectation is, according to Eq. (15):

$$
E_{X}\left[\mathrm{I}_{\infty}(\mathrm{X})\right]=\mathrm{E}_{M, X}\left[\mathbf{1}_{\mathrm{Y}(X)<\Delta}\right]=\frac{1}{\mathrm{~S}} \int_{S} \mathrm{P}(Y<\delta(s)) d s=1-\frac{1}{\mathrm{~S}} \int_{S}\left(1-\lambda_{1} \delta(\mathrm{s})\right)^{\lambda / \lambda_{1}} d s
$$

285 As pointed out above, the variance of I has two components. The conditional variance 286 formula provides:

$$
\begin{aligned}
& \operatorname{Var}(\mathrm{I}(\mathrm{X}))=E_{X} {\left[\operatorname{Var}_{M}(\mathrm{I}(\mathrm{X}))\right]+\operatorname{Var}_{X}\left(E_{M}[\mathrm{I}(\mathrm{X})]\right) } \\
&=E_{X}\left[\operatorname{Var}_{M}\left(\mathrm{I}(\mathrm{X}) \mid \mathrm{I}_{\infty}(X)\right)\right]+\operatorname{Var}_{X}\left(\mathrm{I}_{\infty}(\mathrm{X})\right)
\end{aligned}
$$

287 Now, assuming that the $\mathrm{N}$ beams are independent and identically distributed, one gets

$288 \operatorname{Var}_{M}(\mathrm{I})(X)=\frac{\mathrm{I}_{\infty}(\mathrm{X})\left(1-\mathrm{I}_{\infty}(\mathrm{X})\right)}{\mathrm{N}}$, since I is simply a proportion estimated on a sample of size N.

289 The variance $\operatorname{Var}_{X}\left(\mathrm{I}_{\infty}(X)\right)$ is due to the variability of element positions in a vegetation sample.

290 This variance becomes negligible when the vegetation sample is made of a large number of

291 small area elements, but it cannot be evaluated in closed form for actual configurations of $X$.

292 It is instead approximated by numerical simulations, as described in section 4 (for $\lambda_{1} \bar{\delta}<0.3$ ): 


$$
\operatorname{Var}_{X}\left(\mathrm{I}_{\infty}(X)\right) \approx \sigma_{\mathrm{I}_{\infty}}^{2}\left(\mathrm{I}_{\infty}(X)\right)=0.230 \lambda_{1} \bar{\delta} \mathrm{I}_{\infty}(X)^{1.903-2.30 \lambda_{1} \bar{\delta}}\left(1-\mathrm{I}_{\infty}(X)\right)
$$

293 From now on, we drop the dependence to $X$ and $M$ for the ease of notations. Putting these

294 results together leads to the following estimator for the variance of I:

$$
\sigma_{I}^{2}=\frac{\mathrm{I}(1-\mathrm{I})}{\mathrm{N}}+\sigma_{\mathrm{I}_{\infty}}^{2}\left(\mathrm{I}, \lambda_{1} \bar{\delta}\right)
$$

295 Hence to the following Wald confidence interval for the expectation of the asymptotic RDI, $296 \mathrm{I}_{\infty}$ :

$$
\mathrm{I} \pm z_{\alpha / 2} \sqrt{\frac{\mathrm{I}(1-\mathrm{I})}{\mathrm{N}}+\sigma_{\mathrm{I}_{\infty}}^{2}\left(\mathrm{I}, \lambda_{1} \bar{\delta}\right)}
$$

297 where $z_{\alpha / 2}$ is the usual $1-\frac{\alpha}{2}$ quantile of the standard Gaussian distribution.

298

299 The Wald interval is known to have a lower-than-expected coverage probability when the 300 empirical proportion (here, the RDI) is close to 0 or 1 . It means that the actual value of $\lambda$ is 301 less frequently within the estimated interval than expected (Brown et al., 2001). As an 302 example, it is obviously the case when $\mathrm{I}=0$ (or 1 ), since the true value is supposed to be 0 (or 1) at $100 \%$. This is problematic since both cases are quite frequent in TLS data. Among

304 others, the Agresti-Coull interval is a simple alternative to the Wald interval recommended in 305 Brown et al. (2001). Its formulation is similar to that of the Wald interval:

$$
\mathrm{I}_{c} \pm z_{\alpha / 2} \sqrt{\frac{\mathrm{I}_{c}\left(1-\mathrm{I}_{c}\right)}{\mathrm{N}_{c}}+\sigma_{\mathrm{I}_{\infty}}^{2}\left(\mathrm{I}_{c}, \lambda_{1} \bar{\delta}\right)}
$$

306 with corrected values of "I" and "N" defined as follow:

$$
\left\{\begin{array}{c}
\mathrm{I}_{c}=\frac{\mathrm{I}+\frac{z_{\alpha / 2}^{2}}{2 \mathrm{~N}}}{1+\frac{z_{\alpha / 2}^{2}}{\mathrm{~N}}} \\
\mathrm{~N}_{c}=\mathrm{N}+z_{\alpha / 2}^{2}
\end{array}\right.
$$

307 This correction leads to confidence intervals that are not centered on I and wider than the

308 Wald interval. Agresti and Coull interval is known to have a higher-than-expected coverage 
probability when $\mathrm{N}$ is small, which is not fully satisfactory, but safer than the Wald interval

310 (Brown et al., 2001).

311

\section{3.3. Point and Variance Estimation of the unbiased Beer-Lambert estimator}

313 The Beer-Lambert estimator derives from solving the transmittance of the voxel medium

314 (section 2.1) and thus rely on the empirical relative density index. In supplementary S2, we

315 extend this approach to define the unbiased Beer-Lambert estimator $\hat{\Lambda}$, valid for close-to316 equal path lengths:

$$
\hat{\Lambda}= \begin{cases}-\frac{1}{\overline{\delta_{e}}}\left(\log (1-I)+\frac{I}{2 N(1-I)}\right) & \text { when } I<1 \\ \frac{\log (2 N+2)}{\overline{\delta_{e}}} & \text { when } I=1\end{cases}
$$

317 with the mean "effective" path length:

$$
\overline{\delta_{e}}=\operatorname{mean}\left(-\frac{\log \left(1-\lambda_{1} \delta_{\mathrm{j}}\right)}{\lambda_{1}}\right)
$$

318 When $\lambda_{1} \ll \frac{1}{\bar{\delta}}$, it simplifies to $\overline{\delta_{e}} \approx \bar{\delta}$ (mean path length).

320 The first term in Eq. (27) when $\mathrm{I}<1$ accounts for the size of elements (Eq. (11)). The second term is a bias correction for the instrument sampling, that compensates a systematic bias caused by the convexity of the log function (See Supplementary S2 for details). Such a bias has never been reported before, to the best of our knowledge.

324 When $\mathrm{I}=1$, the above formulation is derived from the application of the Beer-Lambert law to

325 the center of the Agresti-Coull interval, which is more robust than I.

327 For unequal path lengths, the transmittance equation can be approximated as a second order 328 polynomial in $\lambda$, which leads to the following unbiased estimator: 


$$
\hat{\Lambda}_{2}=\frac{1}{a_{e}}\left(1-\sqrt{1-2 a_{e} \hat{\Lambda}}\right)
$$

329 with $\hat{\Lambda}$ in Eq. (27) and $a_{e}$ the ratio between empirical variance to mean of the effective path 330 length:

$$
a_{e}=\operatorname{var}\left(-\frac{\log \left(1-\lambda_{1} \delta_{\mathrm{j}}\right)}{\lambda_{1}}\right) / \operatorname{mean}\left(-\frac{\log \left(1-\lambda_{1} \delta_{\mathrm{j}}\right)}{\lambda_{1}}\right)
$$

331 Notice that when $\lambda_{1} \ll \frac{1}{\bar{\delta}}, \mathrm{a}_{e} \approx \frac{\sigma_{\delta}^{2}}{\bar{\delta}}$, with $\sigma_{\delta}^{2}$ the empirical variance of path lengths.

333 The variances of both unbiased estimators $\widehat{\Lambda}$ and $\widehat{\Lambda}_{2}$ can be derived from the variance of the 334 RDI (Eq. 23), as shown in Supplementary S2:

$$
\sigma_{\widehat{\Lambda}}^{2}= \begin{cases}\frac{1}{{\overline{\delta_{e}}}^{2}}\left(\frac{I}{N(1-I)}+\frac{\sigma_{\mathrm{I}_{\infty}}^{2}\left(\mathrm{I}, \lambda_{1} \bar{\delta}\right)}{(1-I)^{2}}\right)\left(1-\frac{1}{2 N(1-I)}\right)^{2} & \text { when } I<1 \\ \frac{1}{{\overline{\delta_{e}}}^{2}}\left(2+\frac{1}{N}+(2 N+2)^{2} \sigma_{\mathrm{I}_{\infty}}^{2}\left(\frac{2 N+1}{2 N+2}, \lambda_{1} \bar{\delta}\right)\right) & \text { when } I=1\end{cases}
$$

335 and

$$
\sigma_{\widehat{\Lambda}_{2}}^{2}=\sigma_{\widehat{\Lambda}}^{2}\left(1+2 \mathrm{a}_{e} \hat{\Lambda}+4\left(\mathrm{a}_{e} \hat{\Lambda}\right)^{2}\right)
$$

336 These estimators account for the instrument sampling (with the $1 / \mathrm{N}$ term when $\mathrm{I}<1$ ), the asymptotic variability of element positions between vegetation samples (terms with $\sigma_{\mathrm{I}_{\infty}}^{2}$ ) and

338 the convexity of the log function (third factor when $\mathrm{I}<1$ ). As above, the case corresponding to

$339 \mathrm{I}=1$ is based on the center of the Agresti-Coull interval.

\subsection{Point and Variance Estimation from Maximum Likelihood Estimation}

342 The following estimator is derived from Maximum Likelihood (Kay, 1993, Chapter 7), that

343 uses the full information provided by the TLS, namely the actual distribution of free paths in

344 the voxel $\left\{z_{j}\right\}_{j=1, N}$ the $\mathrm{N}$ free paths. In supplementary S3, we compute the analytical MLE 345 from the expected free path distribution (Eq. (17)). These derivations show that the modified 
contact frequency proposed in Béland et al. (2011) is indeed the MLE, which demonstrates its asymptotic consistency when $\mathrm{N}$ is large. This formulation is extended to the case of elements

348 of finite size, thanks to the "effective" mean free path:

$$
\overline{\mathrm{z}_{e}}=\operatorname{mean}\left(-\frac{\log \left(1-\lambda_{1} \mathrm{z}_{\mathrm{j}}\right)}{\lambda_{1}}\right)
$$

349 The MLE is asymptotically-normal, meaning that its residuals become normal when $\mathrm{N}$ is

350 large (Kai, 1993). However, the MLE is biased when the number of beams is finite. In

351 Supplementary S3, we account for this bias, which leads to the following unbiased MLE:

$$
\widetilde{\Lambda}=\frac{\mathrm{I}}{\overline{\mathrm{z}_{e}}}-\frac{\overline{\mathbf{1}_{\mathrm{z}<\delta} z_{e}}}{\mathrm{~N}_{\bar{e}}^{2}}
$$

352 with

$$
\overline{\mathbf{1}_{\mathbf{z}<\delta^{Z_{e}}}}=\operatorname{mean}\left(-\frac{\mathbf{1}_{\mathrm{z}_{\mathrm{j}}<\delta_{\mathrm{j}}} \log \left(1-\lambda_{1} \mathrm{z}_{\mathrm{j}}\right)}{\lambda_{1}}\right)
$$

353 Compared to the Beer-Lambert estimator, this approach does not require any correction for 354 unequal path lengths.

356 In supplementary S3, we rigorously compute the variance of $\widetilde{\Lambda}$ with bias correction for

357 instrument sampling and the variability of element positions between vegetation samples:

$$
\sigma_{\widetilde{\Lambda}}^{2}=\frac{\mathrm{I}}{\mathrm{Nz}_{e}{ }^{2}}\left(1-\frac{\overline{\mathbf{1}_{\mathrm{z}<\delta} z_{e}}}{N I \overline{z_{e}}}\right)^{2}+\frac{\sigma_{I_{\infty}}^{2}\left(\mathrm{I}_{\mathrm{b}}, \lambda_{1} \bar{\delta}\right)}{\bar{\delta}_{e}^{2}\left(1-\mathrm{I}_{\mathrm{b}}\right)^{2}}
$$

358 With

$$
\mathrm{I}_{\mathrm{b}}=\min \left(\mathrm{I}, 1-\frac{1}{2 N+2}\right)
$$

359 The factor involving $\overline{\mathbf{1}_{\mathbf{z}<\delta^{Z_{e}}}}$ expresses the bias correction for the instrument sampling and the

360 term with $\sigma_{I_{\infty}}^{2}$ derives from the variability of vegetation samples, as for the variance of the

361 unbiased Beer-Lambert estimator. 


\subsection{Cramer-Rao bound for variance}

364 The Cramer-Rao bound is the theoretical lower bound of the variance of unbiased estimators

365 (Kay, 1993, Chapter 3), meaning that an unbiased estimator with variance as small as the

366 Cramer-Rao bound is optimal. In Supplementary S3, we show that when the vegetation

367 samples are not fixed (i.e. when TLS beams are shot on variable vegetation samples), the

368 Cramer-Rao bound is:

$$
\mathrm{CRB}_{\lambda}=\frac{\lambda^{2}}{\mathrm{NI}_{\infty}(\lambda)}
$$

369 This analytical formulation is helpful, since the value of the Cramer-Rao bound can be

370 analytically computed (integrating Eq. (20) to compute $I_{\infty}(\lambda)$ ), when both attenuation

371 coefficient and voxel geometry are known. This is the case in the numerical simulations

372 developed in sections 4 and 5, which thus provides a way to evaluate the efficiency of the

373 unbiased estimators, that are expected to have empirical variances as close as possible to

$374 \mathrm{CRB}_{\lambda}$. A perfect match corresponds to the most efficient estimator.

375 It is important to notice however, that this theoretical bound can never be achieved when the

376 variability of vegetation samples has a significant contribution to the variance of the RDI,

377 since this variability is not accounted for in this theoretical bound (see Supplementary S3 for

378 more details).

379

$380 \quad$ 3.6. Estimating confidence intervals for a voxel or a group of voxel

381 From unbiased estimators $\widetilde{\Lambda}$ and $\sigma_{\widetilde{\Lambda}}^{2}$, the confidence interval at a risk level $\alpha$ can naturally be

382 estimated as:

$$
\tilde{\Lambda} \pm z_{\alpha / 2} \sqrt{\sigma_{\widetilde{\Lambda}}^{2}}
$$

383 However, such a formulation is expected to have the same limitations as the Wald interval for

384 the RDI, when the probabilities of interception (RDI) are low or high. This interval is thus 
385

386

387 estimation of $\widetilde{\Lambda}$ and $\sigma_{\widetilde{\Lambda}}^{2}$, leading to:

$$
\tilde{\Lambda}_{c} \pm z_{\alpha / 2} \sqrt{\sigma_{\tilde{\Lambda}_{c}}^{2}}
$$

388 If estimations at voxel scale is a key outcome of TLS, the scale of interest is often larger, 389 typically the individual plants, the horizontal slice of vegetation, or the forestry plot. In this

390 case, the variable of interest is not the single voxel estimation, but the average attenuation

391 coefficient in a group of voxels. For a group of $n_{v}$ voxels and assuming independence between

392 voxels, the confidence interval on the mean of attenuation coefficient estimators is:

$$
\frac{1}{n_{v}} \sum_{n_{v}} \tilde{\Lambda} \pm \frac{z_{\alpha / 2}}{n_{v}} \sqrt{\sum_{n_{v}} \sigma_{\widehat{\Lambda}^{2}}^{2}}
$$

393 The $95 \%$ errors, defined as the radius of the confidence interval at $95 \%$, for a single voxel or a

394 group of voxel are thus:

$$
E 95_{\widetilde{\Lambda}}=1.96 \sigma_{\widetilde{\Lambda}}
$$

395 And

$$
E 95_{\widetilde{\Lambda}}^{n_{v}}=\frac{1.96}{n_{v}} \sqrt{\sum_{n_{v}} \sigma_{\widetilde{\Lambda}}^{2}}
$$

396 Similar quantities can be defined for the unbiased Beer-Lambert estimators $\widehat{\Lambda}$ and $\widehat{\Lambda}_{2}$, as well

397 as the bound of the $95 \%$ error, based on the Cramer-Rao bound for variance, which is the 398 lower bound of $95 \%$ error for an unbiased estimator (i.e. no unbiased estimator can lead to 399 smaller errors).

400 


\section{$401 \quad$ 4. Design of the numerical experiments}

402

403

\subsection{Overview}

404 The aim of our numerical simulations is to compare the estimates of attenuation coefficients

405 to their true values. Simple configurations are generated to simulate replicates of virtual TLS

406 point clouds in voxels filled with idealized vegetation of known properties. The numerous

407 replicates enable to compute various statistics through a MonteCarlo approach, to evaluate the

408 consistency and efficiency of each estimator, as well as the consistency of variance and

409 confidence interval estimators. For each estimator, we compute its expectation, variance and

$41095 \%$ errors. The $95 \%$ errors are estimated as the $95^{\text {th }}$ percentile of the absolute residuals and

411 are evaluated for a single voxel and a group of voxels (here $n_{v}=100$ ). We also compute the

412 expectation of variance estimators, as well as the coverage probability of the estimated

413 confident intervals, which is the empirical frequency at the true value of the attenuation

414 coefficient belongs to the estimated confidence interval.

415 These statistics are computed for various values of attenuation coefficients, element sizes,

416 voxel sizes, and beam numbers. Simulations are run for two different configurations described

417 below. The first configuration, described in details in subsection 4.2, assumes finite element

418 size (meaning that $\lambda_{1}>0$ ) and equal path lengths. Equal path lengths imply that the second

419 order correction for the Beer-Lambert estimators (i.e. $\widehat{\Lambda}^{2}$ ) is not required. The computation of

420 RDI and distance travelled is done over the actual distributions of vegetation elements

421 (generated with random positions), each corresponding to a fixed vegetation sample $X$.

422 Simulations are run for a large number of vegetation samples, which entails to compute the

423 asymptotic variance of the RDI, $\sigma_{I_{\infty}}^{2}$, (when the beam number is infinite) for each value of

424 attenuation coefficient and element size. The second set of simulations (subsection 4.3) is

425 specifically designed to evaluate the correction for unequal path lengths involved in unbiased 
426 Beer-Lambert estimator $\widehat{\Lambda}_{2}$. For simplicity, we assume that vegetation elements are infinitely

427 small $\left(\lambda_{1}=0\right)$ and that the voxel is a sphere, so that the free-path distribution and the 428 Cramer-Rao bound can be analytically solved (See Supplementary S4 for details). We 429 separate both sets of simulations to facilitate the presentation of results.

430 In order to simplify the numerical experiment design and the presentation of the results, we 431 build dimensionless quantities, namely the beam depth $\left(\mathrm{L}_{\mathrm{j}}=\lambda \delta_{\mathrm{j}}\right)$ and the voxel depth $(\mathrm{L}=$ $432 \lambda \bar{\delta})$. Likewise, $y_{j}=\lambda z_{j}$ is the free depth for beam $j$. We can notice that I can be computed

433 from the distribution of depth $\left\{\mathrm{y}_{\mathrm{j}}\right\}$ since $\mathbf{1}\left(\mathrm{z}_{\mathrm{j}}<\delta_{\mathrm{j}}\right)=\mathbf{1}\left(\mathrm{y}_{\mathrm{j}}<\mathrm{L}_{\mathrm{j}}\right)$. The element depth is $\mathrm{L}_{1}=$ $434 \lambda_{1} \bar{\delta}$. More generally, dimensionless quantities can be derived for all quantities of interest 435 developed above, as shown in Supplementary S5. The practical interest of this substitution is 436 that computations can be done for a series of voxel depth values, and easily extrapolated to $\lambda$ 437 by simply dividing results by $\bar{\delta}$, instead of running simulations for series of $(\lambda, \bar{\delta})$ values.

\subsection{Numerical simulations for finite-size elements and equal path lengths}

440 Simulated vegetation samples correspond to flat square elements that are randomly distributed 441 in a voxel, parallel to one face of the voxel. The virtual beams are sent perpendicular to 442 elements, so that the path lengths are equal to cube size (Figure 1). The voxel and element 443 depths are $\mathrm{L}=\lambda \delta$ and $\mathrm{L}_{1}=\lambda_{1} \delta$, respectively. 
445

Table 1. Parameter values in numerical simulations

\begin{tabular}{|l|l|}
\hline Parameter & Values \\
\hline Voxel depth $(\mathrm{L})$ & $0.05,0.1,0.5,1,1.5,2,2.5,3$ \\
\hline Beam number $(\mathrm{N})$ & $3,5,7,10,15,20,30,40,50,75,100,150,200,300,400,500,7501000,5000,10000$ \\
\hline Element depth $\left(\mathrm{L}_{1}\right)$ & $\begin{array}{l}\text { Subsection 4.2: } 0.001,0.005,0.01,0.05,0.1,0.2,0.3,0.5 \\
\text { Subsection 4.3: } 0\end{array}$ \\
\hline
\end{tabular}

$\mathrm{Nb}$ : in subsection 4.3 , elements are assumed infinitely small so that $\mathrm{L}_{1}=0$.

Fig. 1. Illustration of a numerical simulation of TLS beams over finite-size elements and equal path length $\delta$. Each square element has a size equal to $\sqrt{\mathrm{S}_{1}}=\sqrt{\lambda_{1} \delta^{3}}=\sqrt{\mathrm{L}_{1}} \delta$.

For each value of $L$ and $L_{1}$ tested (Table 1), we simulate 10000 vegetation samples on which we shoot $\mathrm{M}$ batches of $\mathrm{N}$ virtual beams, with $\mathrm{N}$ between 5 and 10000 . Batches serve as replicates of TLS shooting, to compute the different statistics (estimator expectation, variance, $95 \%$ error, variance and confidence interval estimator, confidence interval coverage probability). The batch numbers are selected so that the total number of beams $\mathrm{MN}$ is constant, equal to $=10^{8}$. This number is large enough for the convergence of the different statistics, despite the replicate number $\mathrm{M}$ decreases with $\mathrm{N}$, since the variance of the estimates sharply decays with $\mathrm{N}$.

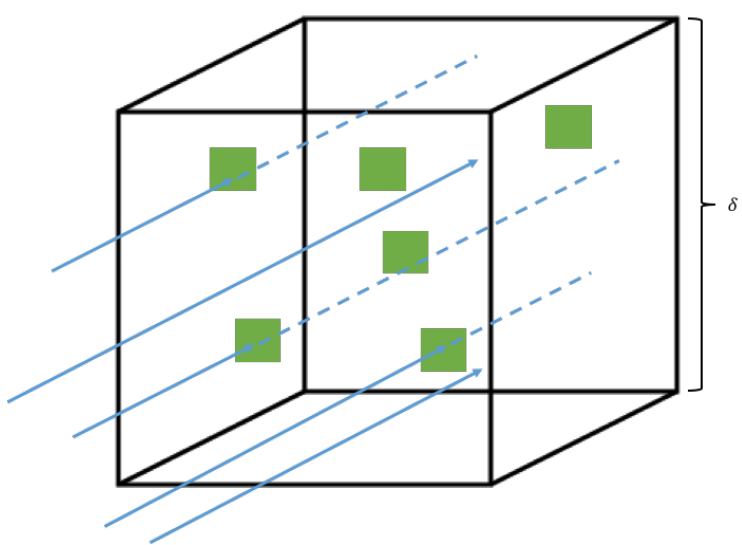


461 We use simulations with the largest beam number $(N=10000 \approx \infty)$ to estimate the 462 asymptotic variance $\sigma_{\mathrm{I}_{\infty}}^{2}$, for various values of $\mathrm{L}_{1}$ and $\mathrm{L}$. We remind that this variance is due 463 to the variability of element position between vegetation samples $X$. When $\mathrm{L}_{1}<0.3$, which is 464 the case for most vegetation when voxels are greater than $10 \mathrm{~cm}$ (See Supplementary S1), $465 \sigma_{\mathrm{I}_{\infty}}^{2}$ can be estimated from a simple empirical function of $\mathrm{L}_{1}$ and RDI (Fig. 2 and Eq. (22)).

466

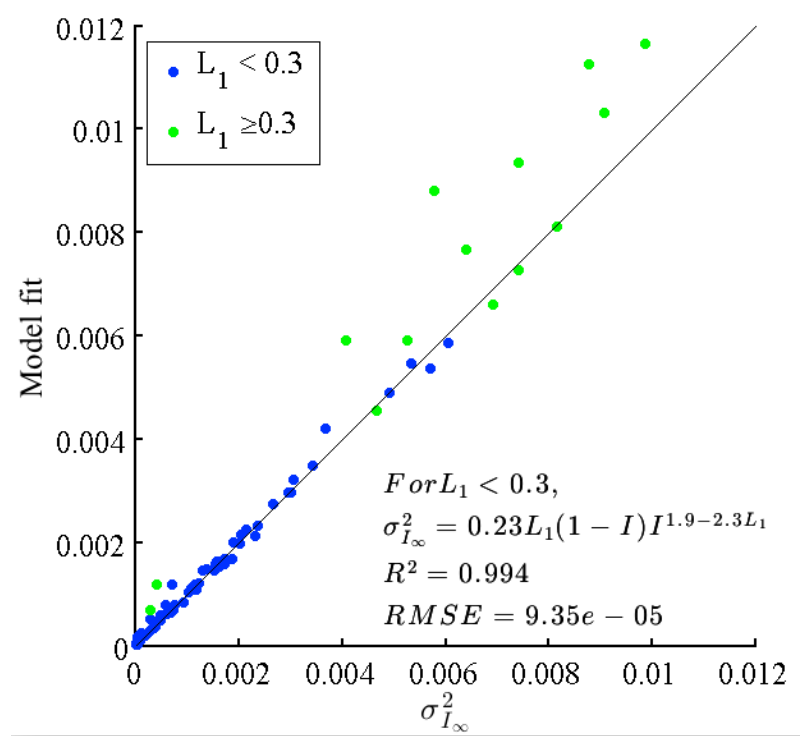

467 Fig. 2. Empirical model for the variance of the asymptotic relative density index $\mathrm{I}_{\infty} . \sigma_{\mathrm{I}_{\infty}}^{2}$ is caused by

468 the variability of element positions between vegetation samples.

469

\subsection{Numerical simulations for unequal path lengths}

471 In a sphere with radius $\mathrm{R}$, the voxel depth is:

$$
\mathrm{L}=\lambda \bar{\delta}=\lambda \frac{\mathrm{V}}{\mathrm{S}}=\lambda \frac{\frac{4}{3} \pi \mathrm{R}^{3}}{\pi \mathrm{R}^{2}}=\frac{4}{3} \lambda \mathrm{R}
$$

472 The distribution of dimensionless optical depth $L_{j}$ is, with $u$ between 0 and 1 (Supplementary 473 S4):

$$
\operatorname{PDF}\left(\mathrm{L}_{\mathrm{j}}=\frac{3}{2} \mathrm{~L} \sqrt{1-\mathrm{u}^{2}}\right)=2 \mathrm{u}
$$


474 For each value of $\mathrm{L}$ (Table 1), we simulate a total of $10^{8}$ virtual beams with lengths $\left\{\mathrm{L}_{\mathrm{j}}\right\}$ and

475 the corresponding free path lengths $\left\{y_{j}\right\}$, that respectively follow Eq. (45) and the exponential 476 law. As in subsection 4.2, virtual beams are grouped in batches of $\mathrm{N}$ beams to simulate $\mathrm{M}$ 477 replicates of TLS shooting and to compute the same statistics as above.

478

\section{5. Numerical simulation results}

480 In this section, we show the statistics described in section 4. Subsection 5.1. corresponds to 481 finite size element simulations (described in subsection 4.2), whereas subsection 5.2. 482 corresponds to unequal path simulation (described in subsection 4.3). Expectation, variance 483 and $95 \%$ error enable to compare the consistency and efficiency of the usual estimators $(\hat{\lambda}$ and $484 \tilde{\lambda})$ and the new ones $\left(\widehat{\Lambda}, \widehat{\Lambda}_{2}\right.$ and $\left.\widetilde{\Lambda}\right)$. We also show the expectation of the variance estimators $485\left(\sigma_{\widehat{\Lambda}}^{2}, \sigma_{\widehat{\Lambda}_{2}}^{2}\right.$ and $\left.\sigma_{\widetilde{\Lambda}}^{2}\right)$, the confidence interval radiuses $\left(E 95_{\widehat{\Lambda}}, E 95_{\widehat{\Lambda}_{2}}\right.$ and $\left.E 95_{\widetilde{\Lambda}}\right)$, and the coverage 486 probabilities of estimated confidence intervals.

\subsection{Estimator performance for finite size elements}

\subsubsection{Estimator consistency}

491 Figure 3 shows the expectation of estimator derived from the MLE, as a function of the beam 492 number. Blue dots corresponds to the modified contact frequency estimator $\tilde{\lambda}$ (Eq. 8), which is 493 the biased MLE for infinitely small elements. Green dots corresponds to the unbiased MLE $\tilde{\Lambda}$ 494 (Eq. 34), that accounts for element size and beam number. Since expectations are normalized 495 by the true value of $\lambda$, estimators are consistent when expectations equal one, and deviations 496 from 1 quantifies the bias. Subplots $A, B$ and $C$ correspond to small elements $\left(\mathrm{L}_{1}=0.001\right)$ for 497 three voxel depths L, whereas subplots D, E and F correspond to "larger" elements compared to voxel size $\left(\mathrm{L}_{1}=0.1\right)$ for the same values of voxel depths. 
Peer-reviewed version available at Remote Sensing of Environment 2018, 215, 342-370; doi:10.1016/i.rse.2018.06.024

499 Even when elements are small, the modified contact frequency is positively biased when $\mathrm{N}$ is

500 small (subplots A, B, C). Vertical blue lines show the thresholds of $\mathrm{N}$ for which the bias of $\tilde{\lambda}$

501 is larger than $1 \%$. These thresholds range between $\mathrm{N}=30$ and $\mathrm{N}=75$, depending on $\mathrm{L}$. When

502 elements are larger (subplots D, E, F), the positive biases remain for large values of N, so that

503 the $1 \%$ threshold is not reached. On the contrary, the unbiased MLE $\widetilde{\Lambda}$ (green dots) shows

504 biases always lower than 1\% (green lines) when elements are small, even when $\mathrm{N}$ is as small

505 as 3 for $\mathrm{L}_{1}=0.001$ (subplots $\mathrm{A}, \mathrm{B}, \mathrm{C}$ ) and 5 when $\mathrm{L}_{1}=0.1$ (subplots $\mathrm{D}, \mathrm{E}, \mathrm{F}$ ).
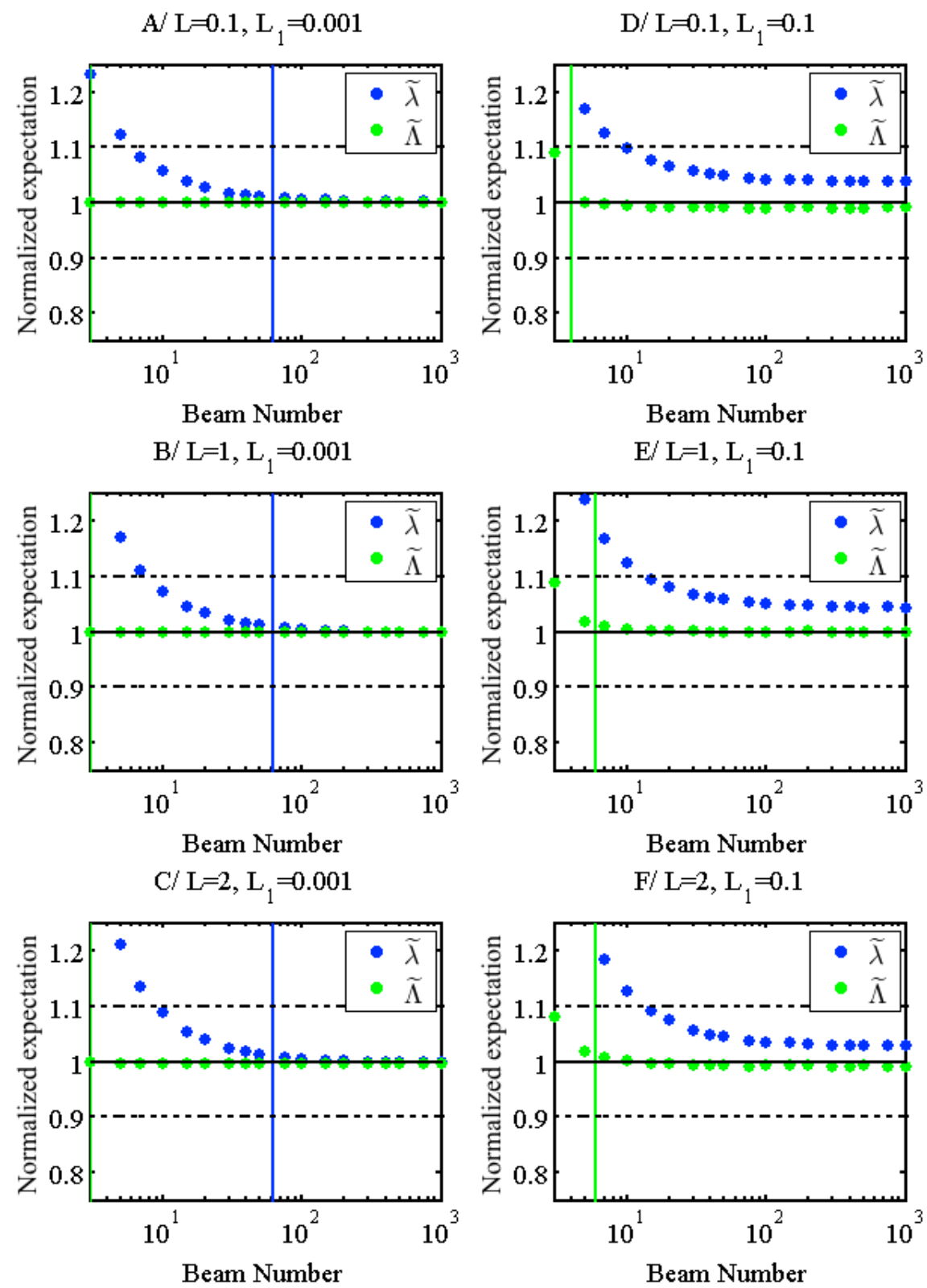
507

508

509

510

511

512

513

514

515

516

517

518

519

520

521

522

523

524

525

526

527

528

529

530

Fig. 3. Expectations of the attenuation coefficient estimators derived from the MLE, as a function of the beam number. Blue dots corresponds to the modified contact frequency estimator $\tilde{\lambda}$ (Eq. 8), which is the biased MLE for infinitely small elements. Green dots corresponds to the unbiased MLE $\widetilde{\Lambda}$ (Eq. 34), that accounts for element size and beam number. Estimators are normalized by their true value $\lambda$, so that they are consistent when the expectation equals to one. The vertical lines correspond to the lowest values of $\mathrm{N}$ leading to a bias smaller than $1 \%$ in blue and green for respectively the biased and unbiased estimators.

Figure 4 is similar to figure 3, but for the usual $(\hat{\lambda})$ and unbiased $(\widehat{\Lambda})$ Beer-Lambert estimators. Trends are similar with two main differences. First, the biases are slightly larger with BeerLambert estimators than with the MLE when the vegetation density is low to moderate $(\mathrm{L}<=1$, subplot A, B, D and E), whether bias are corrected or not. Second, the bias of the usual BeerLambert estimator decreases until becoming negative for small values of $\mathrm{N}$ when the vegetation density is high (subplots C and F). Such a decay is attributed to the occurrence of cases in which all beams are intercepted inside the voxel $(\mathrm{I}=1)$, referred to as "occluded" in Béland et al. (2014a). In this cases, the usual Beer-Lambert estimator is $+\infty$, but is ignored in expectation computation to avoid divergence. Attenuation coefficient estimates are thus bounded by $\frac{\log (\mathrm{N})}{\bar{\delta}}$, leading to this negative bias of increasing magnitude when $\mathrm{N}$ is small. This trend is also visible for $\widehat{\Lambda}$, but it is far less pronounced and it occurs for lower values of $\mathrm{N}$. This demonstrates the benefit of the definition of $\widehat{\Lambda}$, which is extended when $\mathrm{I}=1$ with Agresti-Coull interval centers. The range of consistency of $\widehat{\Lambda}$, however, is clearly narrower than the one of the unbiased MLE $\widetilde{\Lambda}$.

(1)



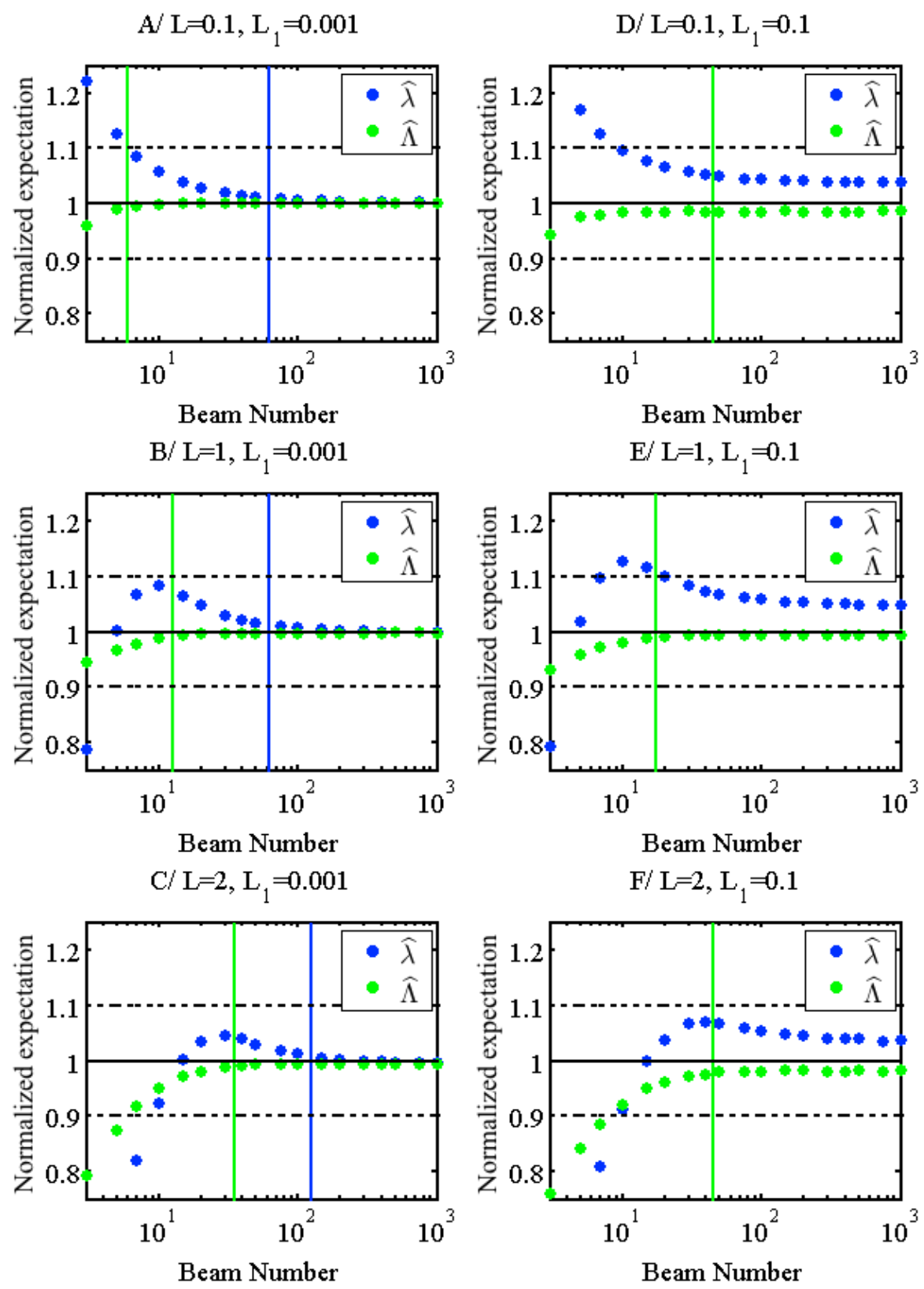

532 Fig. 4. Same as Figure 3 for the usual and unbiased Beer-Lambert estimators.

533 Expectations of the Beer-Lambert-attenuation-coefficient estimators, as a function of the beam

534 number. Blue dots corresponds the usual Beer-Lambert estimator $(\hat{\lambda})$. Green dots corresponds to the

535 unbiased Beer-Lambert estimator $(\widehat{\Lambda})$, that accounts for element size and beam number and extended

536 definition when $\mathrm{I}=1$. Estimators are normalized by their true value $\lambda$, so that they are consistent when

537 the expectation equals to one. The vertical lines correspond to the lowest values of $\mathrm{N}$ leading to a bias

538 smaller than $1 \%$ in blue and green, for respectively the biased and unbiased estimators. 
540 The computations of biases are done for all values of parameters in Table 1 and lead to the

541 range of validity for three levels of consistency $(1 \%, 5 \%, 10 \%)$ for the four estimators

542 summarized in Table 2. As expected from Figures 3 and 4, the usual Beer-Lambert and

543 modified contact frequency are biased in a much wider range than the corrected indices

544 introduced in section 3, and generally requires smaller elements and a larger beam number to

545 be consistent. Also, the biases of the Beer-Lambert estimators increase with density, which

546 leads to less straightforward formulations of range of consistency, since their definition vary

547 with both $\mathrm{L}_{1}$ and L. MLE thus has wider range of validity than Beer-Lambert estimators.

Table 2. Range of consistency of the four estimators of attenuation coefficient for three consistency

\begin{tabular}{|c|c|c|c|}
\hline $\begin{array}{l}\text { Inde } \\
\mathrm{x}\end{array}$ & Consistency (1\%) & Consistency $(5 \%)$ & Consistency $(10 \%)$ \\
\hline$\tilde{\lambda}$ & $L_{1} \leq 0.01$ and $\mathrm{N} \geq 100$ & $\left\{\begin{array}{l}L_{1} \leq 0.01 \text { and } N \geq 20 \\
L_{1} \leq 0.05 \text { and } N \geq 30\end{array}\right.$ & $\left\{\begin{array}{c}L_{1} \leq 0.01 \text { and } \mathrm{N} \geq 10 \\
L_{1} \leq 0.1 \text { and } \mathrm{N} \geq 20\end{array}\right.$ \\
\hline$\widetilde{\Lambda}$ & $\left\{\begin{array}{l}L_{1} \leq 0.01 \text { and } N \geq 3 \\
L_{1} \leq 0.1 \text { and } N \geq 5 \\
L_{1} \leq 0.2 \text { and } N \geq 15 \\
L_{1} \leq 0.3 \text { and } N \geq 30\end{array}\right.$ & $\left\{\begin{array}{l}L_{1} \leq 0.05 \text { and } \mathrm{N} \geq 3 \\
L_{1} \leq 0.1 \text { and } \mathrm{N} \geq 5 \\
L_{1} \leq 0.3 \text { and } \mathrm{N} \geq 10\end{array}\right.$ & $\left\{\begin{array}{c}L_{1} \leq 0.1 \text { and } \mathrm{N} \geq 3 \\
L_{1} \leq 0.2 \text { and } \mathrm{N} \geq 5 \\
L_{1} \leq 0.3 \text { and } \mathrm{N} \geq 7 \\
L_{1} \leq 0.5 \text { and } \mathrm{N} \geq 10\end{array}\right.$ \\
\hline$\hat{\lambda}$ & $L \leq 2$ and $L_{1} \leq 0.01$ and $N \geq 100$ & $L_{1} \leq 0.05$ and $\mathrm{N} \geq 40$ & $\left\{\begin{array}{c}L \leq 2 \text { and } L_{1} \leq 0.01 \text { and } \mathrm{N} \geq 10 \\
L \leq 2.5 \text { and } L_{1} \leq 0.1 \text { and } \mathrm{N} \geq 20 \\
L \leq 3 \text { and } L_{1} \leq 0.1 \text { and } \mathrm{N} \geq 30\end{array}\right.$ \\
\hline$\widehat{\Lambda}$ & $\left\{\begin{array}{c}L \leq 0.5 \text { and } L_{1} \leq 0.2 \text { and } N \geq 7 \\
L \leq 1 \text { and } L_{1} \leq 0.2 \text { and } N \geq 10 \\
L \leq 1.5 \text { and } L_{1} \leq 0.2 \text { and } N \geq 15 \\
L \leq 2 \text { and } L_{1} \leq 0.05 \text { and } N \geq 40 \\
L \leq 2.5 \text { and } L_{1} \leq 0.005 \text { and } N \geq 7 \\
L \leq 3 \text { and } L_{1} \leq 0.001 \text { and } N \geq 75\end{array}\right.$ & $\left\{\begin{array}{c}L \leq 1 \text { and } L_{1} \leq 0.1 \text { and } N \geq 5 \\
L \leq 1.5 \text { and } L_{1} \leq 0.2 \text { and } N \geq 10 \\
L \leq 2 \text { and } L_{1} \leq 0.2 \text { and } N \geq 15 \\
L \leq 2.5 \text { and } L_{1} \leq 0.2 \text { and } N \geq 40 \\
L \leq 3 \text { and } L_{1} \leq 0.1 \text { and } N \geq 75\end{array}\right.$ & $\left\{\begin{array}{c}L \leq 1.5 \text { and } L_{1} \leq 0.2 \text { and } N \geq 5 \\
L \leq 2 \text { and } L_{1} \leq 0.3 \text { and } N \geq 10 \\
L \leq 2.5 \text { and } L_{1} \leq 0.3 \text { and } N \geq 20 \\
L \leq 3 \text { and } L_{1} \leq 0.3 \text { and } N \geq 40\end{array}\right.$ \\
\hline
\end{tabular}

\subsubsection{Estimator efficiency}


555 Figure 5 shows the empirical variances (multiplied by $\delta^{2}$ for the generality of results) of 556 estimator derived from the MLE, similarly to Figure 3. As expected, the variances decay with

557 the beam number. When the elements are large and the density is moderate to high (subplots

558 E and F), variances remain significantly larger than zero, even for large beam numbers, 559 because of the variability between vegetation samples. The variances of the biased and 560 unbiased estimators are similar in magnitude, the former being slightly larger when the beam 561 number is small. Both variances are very close to the theoretical Cramer-Rao bound (in

562 black), when $\mathrm{L}_{1}$ is small (subplots $\mathrm{A}, \mathrm{B}$ and $\mathrm{C}$ ). Since $\widetilde{\Lambda}$ is unbiased when $\mathrm{L}_{1}$ is small, it can 563 thus be considered as efficient. When the elements are large and the vegetation is dense 564 (subplots $\mathrm{E}$ and $\mathrm{F}$ ), the variance of $\widetilde{\Lambda}$ is much larger than the Cramer-Rao bound, even when $\mathrm{N}$ 565 is large. This is because the Cramer-Rao bound does not account for asymptotic variability 566 due to the variability of vegetation samples.

567 The green dashed lines corresponds to the expectation of the estimator of the variance of $\widetilde{\Lambda}$, 568 namely $\sigma_{\widetilde{\Lambda}}^{2}$ (Eq. 36). Its expectation is very close to the empirical expectation of the variance 569 of $\widetilde{\Lambda}$ (green dots), demonstrating the consistency of our variance estimator when the beam 570 number is larger than 5. 

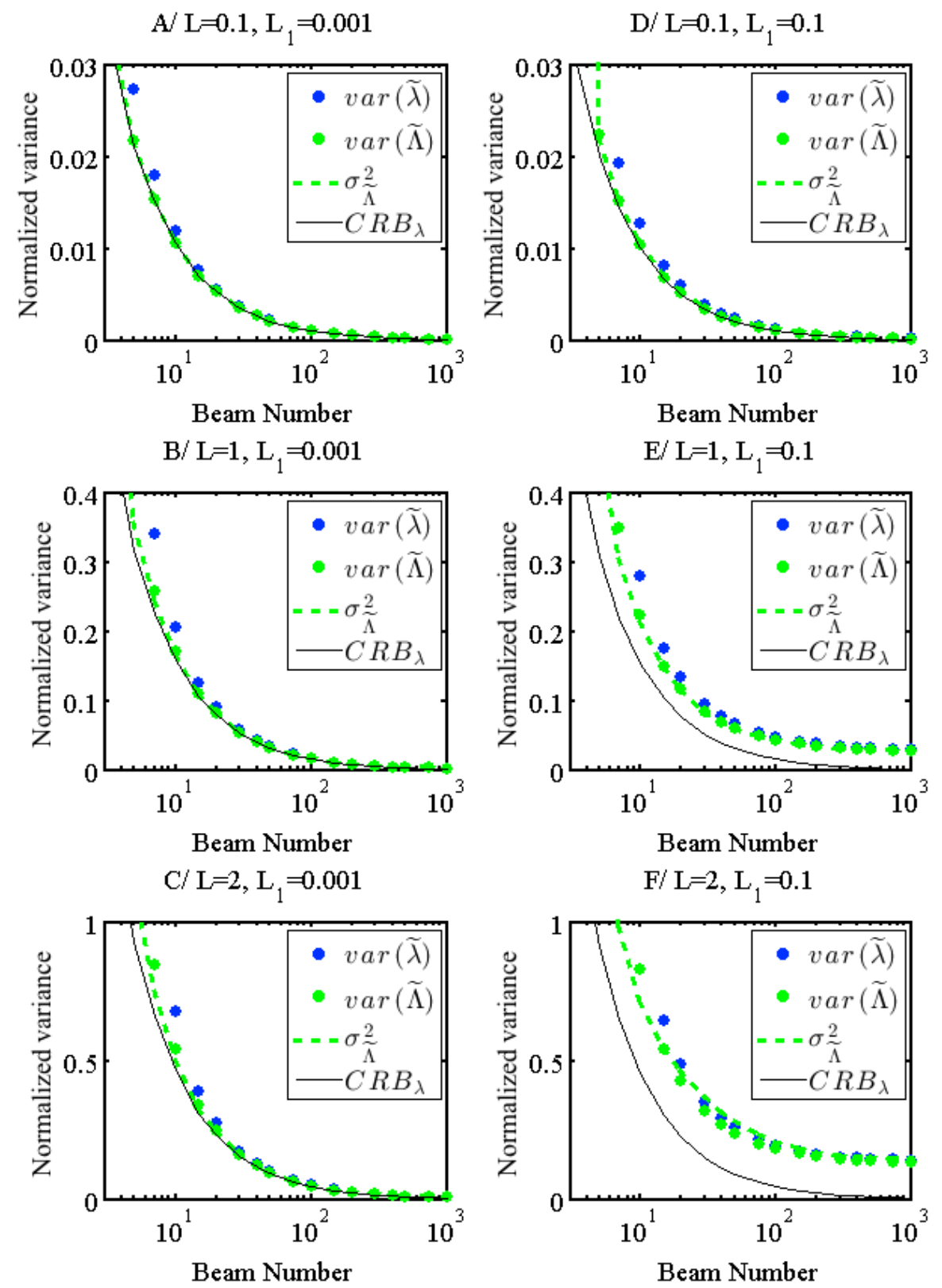

573 Fig. 5. Normalized variances of attenuation coefficient estimators derived from the MLE, as a function

574 of the beam number. Normalized variances correspond to variances multiplied by $\delta^{2}$. Blue dots

575 corresponds to the variance of the modified contact frequency estimator $\tilde{\lambda}$. Green dots corresponds to

576 the variance of the unbiased MLE $\widetilde{\Lambda}$. Green dashed lines correspond to the dimensionless expectation

577 of the variance estimator $\sigma_{\widetilde{\Lambda}}^{2}$. The black line corresponds to Cramer-Rao bound for the variance of 578 unbiased estimator. 
580 Figure 6 is similar to Figure 5 for the variances of Beer-Lambert estimators. Although trends

581 are similar, it is worth noting that the variance of $\widehat{\Lambda}$ is greater than the Cramer-Rao bound for

582 small elements when vegetation is dense (Fig. 6C), showing that $\widehat{\Lambda}$ is suboptimal and less

583 efficient than the MLE. Also, the expectation of the variance estimator $\sigma_{\widehat{\Lambda}}^{2}$ can significantly

584 overestimate the empirical variance, showing a lack of consistency for this estimator. This

585 situation mostly occurs in range of data where $\widehat{\Lambda}$ itself is biased (dense vegetation, low

586 number of beams). The variance of the basic Beer-Lambert law can often be lower than the

587 Cramer-Rao bound. This is simply another evidence that this estimator is strongly biased (due

588 to the I=1 cases), since it would otherwise be greater than the Cramer-Rao bound. 

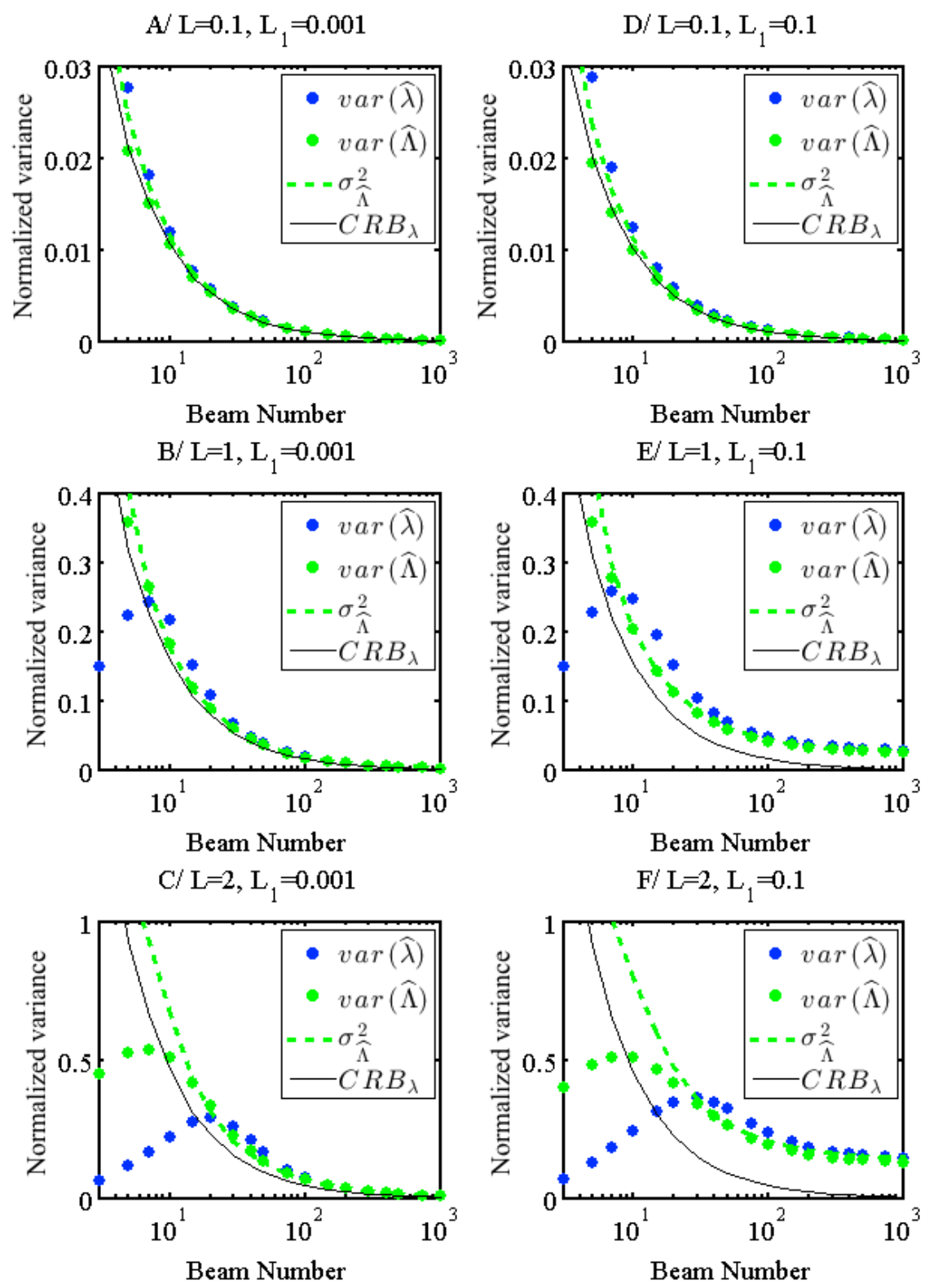

591 Fig. 6. Same as Figure 5 for Beer-Lambert estimators. Normalized variances of Beer-Lambert

592 attenuation coefficient estimators, as a function of the beam number. Normalized variances correspond

593 to variances multiplied by $\delta^{2}$. Blue dots corresponds to the variance of the usual Beer-Lambert

594 estimator $\hat{\lambda}$. Green dots corresponds to the variance of the unbiased Beer-Lambert estimator $\widehat{\Lambda}$. Green

595 dashed lines correspond to the dimensionless expectation of the variance estimator $\sigma_{\widehat{\Lambda}}^{2}$. The black line

596 corresponds to Cramer-Rao bound for the variance of unbiased estimator. 
599 Figure 7 shows the coverage probabilities of the estimated confidence interval based on

600 unbiased MLE, $\widetilde{\Lambda} \pm z_{\alpha / 2} \sqrt{\sigma_{\widetilde{\Lambda}}^{2}}$ for three confidence levels (50, 90 and 95\%). When the 601 confidence intervals are correctly estimated, empirical coverage probabilities should match 602 the confidence level (dashed lines). Estimated confidence intervals are satisfactory in most 603 cases, with the exception of low density when the beam number is low (subplots A and B), for 604 which the true value is less frequently in the confidence interval than expected.

605

606

607

608 

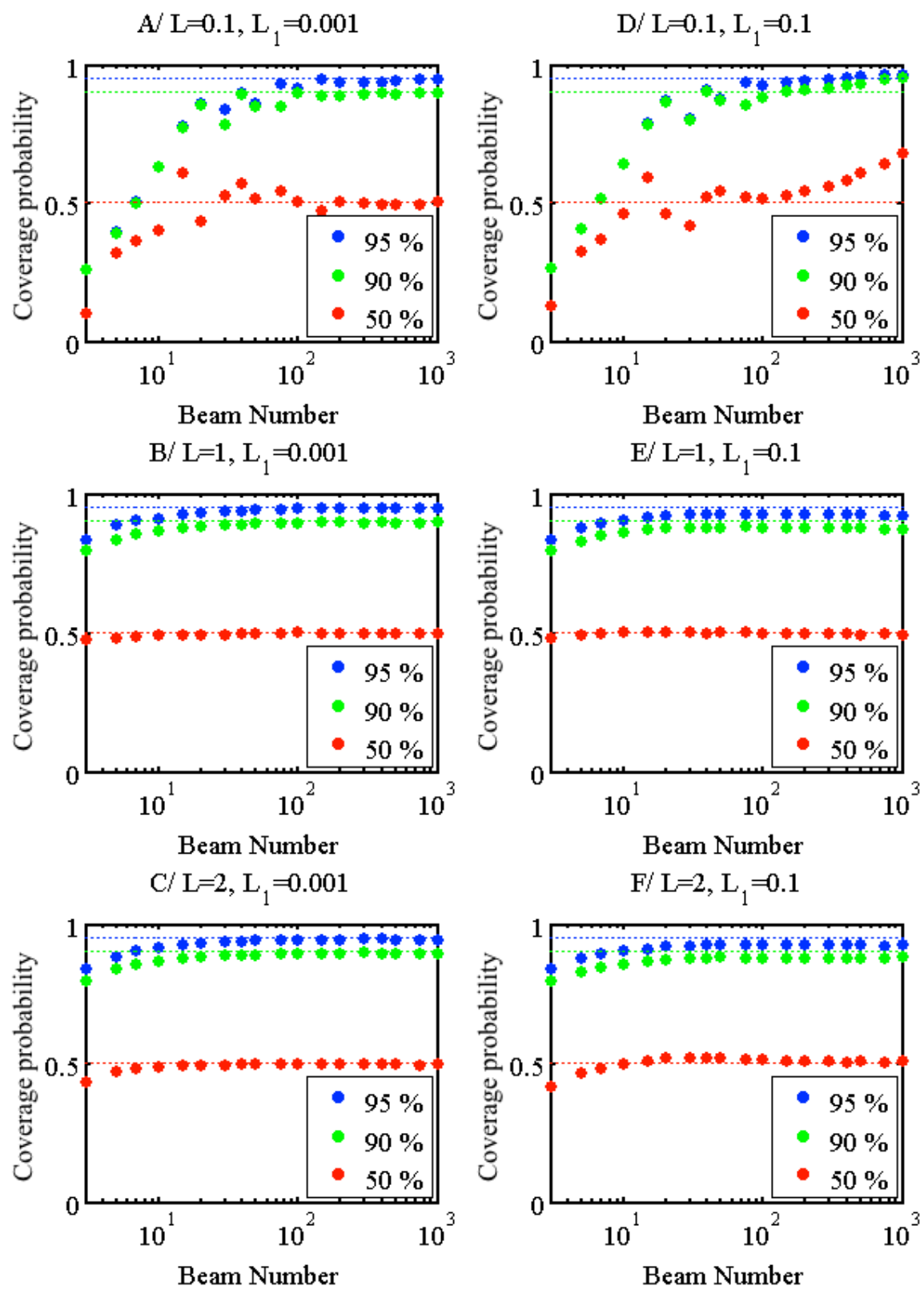

610 Fig. 7. Coverage probabilities of the estimated confidence interval $\widetilde{\Lambda} \pm z_{\alpha / 2} \sqrt{\sigma_{\widetilde{\Lambda}}^{2}}$ (computed with I),

611 function of the beam number, for 3 levels of confidence (50, 90 and 95\%).

612

613 As explained in section 3.5, the alternative interval estimation based on Agresti-Coull 614 correction (Eq. (40)) leads to higher-than-expected coverage rates, as shown in Figure 8,

615 which is safer when density is low. Very similar intervals can be obtained for the unbiased 616 Beer-Lambert $\widehat{\Lambda}$ (not shown). 


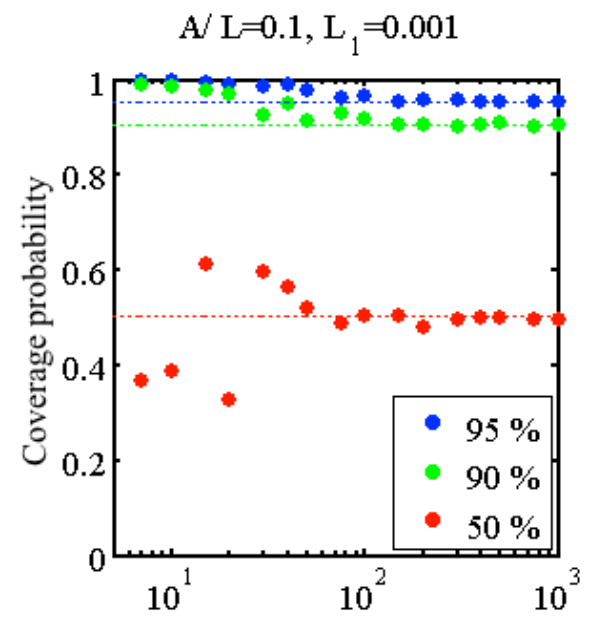

Beam Number

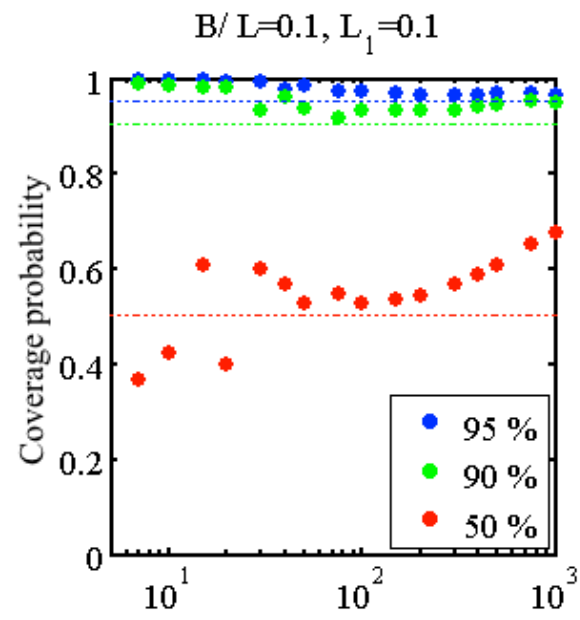

Beam Number

618 Fig. 8. Coverage probabilities of the estimated confidence interval $\widetilde{\Lambda}_{c} \pm z_{\alpha / 2} \sqrt{\sigma_{\widetilde{\Lambda}_{c}}^{2}}$ (based on the

619 Agresti-Coull values $I_{c}$ and $N_{c}$ ), function of the beam number, for 3 confidence levels $(50,90$ and $62095 \%)$

621

622 Coverage probabilities are computed for all simulated cases. For a given confidence level $623(90 \%, 95 \%)$, we can determine the range of parameter values (beam number, element and 624 voxel depths) for which the coverage probabilities match the expected value $(0.9,0.95)$, within $5 \%$ for both the usual formulation of confidence interval (Eq. (39)) and for the 626 "Agresti-Coull" interval (Eq. (40)). We find that using the "Agresti-Coull” interval increases 627 the range of validity, when $\mathrm{L}$ is estimated lower than 0.5 , but that the usual formulation 628 performs better for higher densities. We thus adopt the following partical rule

$$
\left\{\begin{aligned}
\widetilde{\Lambda}_{c} \pm z_{\frac{\alpha}{2}} \sqrt{\sigma_{\widetilde{\Lambda}_{c^{\prime}}}^{2}} & \text { when } \tilde{L} \leq 0.5 \\
\widetilde{\Lambda} \pm z_{\frac{\alpha}{2}} \sqrt{\sigma_{\widetilde{\Lambda}^{\prime}}^{2}}, & \text { when } \tilde{L}>0.5
\end{aligned}\right.
$$

630 We summarize the ranges of validity of confidence intervals defined as in Eq. 46 in Table 3.

631 Confidence intervals are consistent in a fairly large range of parameters. As in the results 632 presented above, the range of validity of the unbiased "Beer-Lambert" confidence interval is 
not as wide as the one of the unbiased MLE, especially when the voxel depth is larger than 2, for which more than 100 beams are required.

Table 3. Range of validity of the confidence intervals at rate $\alpha=0.90$ and 0.95 . We consider that the confidence interval is consistent, when the empirical probability reaches the expected level within 5\%. formulation is used otherwise (Eq. 46).

\begin{tabular}{|c|c|c|}
\hline Index & $\begin{array}{l}\alpha=0.90 \text { and Coverage probability within } \\
0.9 \pm 5 \%\end{array}$ & $\begin{array}{l}\alpha=0.95 \text { and coverage probability within } 0.95 \pm \\
5 \%\end{array}$ \\
\hline$\widetilde{\Lambda}$ & $\left\{\begin{array}{c}L \geq 0.1 \text { and } L_{1} \leq 0.1 \text { and } \mathrm{N} \geq 10 \\
L_{1} \leq 0.1 \text { and } \mathrm{N} \geq 100\end{array}\right.$ & $\left\{\begin{array}{c}L \geq 0.1 \text { and } L_{1} \leq 0.1 \text { and } \mathrm{N} \geq 10 \\
L_{1} \leq 0.1 \text { and } \mathrm{N} \geq 20\end{array}\right.$ \\
\hline$\widehat{\Lambda}$ & $\left\{\begin{array}{c}L \in[0.5 ; 2] \text { and } L_{1} \leq 0.05 \text { and } \mathrm{N} \geq 40 \\
L_{1} \leq 0.01 \text { and } \mathrm{N} \geq 100 \\
L_{1} \leq 0.05 \text { and } \mathrm{N} \geq 200\end{array}\right.$ & $\left\{\begin{array}{c}L \leq 2 \text { and } L_{1} \leq 0.05 \text { and } \mathrm{N} \geq 30 \\
L \leq 2.5 \text { and } L_{1} \leq 0.01 \text { and } \mathrm{N} \geq 100 \\
L_{1} \leq 0.05 \text { and } \mathrm{N} \geq 150\end{array}\right.$ \\
\hline
\end{tabular}

640

\subsubsection{5\% errors for a single voxel and a group of voxels}

Figure 9 shows the expectation of the $95 \%$ errors for the MLE estimators in the same setting as before. When the beam number is small and the density is low, this percentage can largely exceed $100 \%$. In these cases, the estimates remain very uncertain, although close to optimal (Cramer-Rao-95\%-error bound in black). The accuracy increases with vegetation density and

647 beam number. However, the 95\% errors remain very high even for large beam number, when elements are large because of the variability of vegetation samples (subplots $\mathrm{E}$ and F). At the scale of a single voxel, using $\tilde{\lambda}$ or $\widetilde{\Lambda}$ leads to similar errors, which may be disappointing. This is explained by the fact that the bias corrections accounted for in $\widetilde{\Lambda}$ are significant in a range of parameter values for which variances are fairly large. Results are very different, however,

652 when errors are computed after averaging over several voxels (here, 100 voxels), which leads 
654 modified contact frequency $(\tilde{\lambda})$ leads to a reduction of the error on the order of $50 \%$, typically

655 in cases with low beam number or large elements, demonstrating the interest of bias 656 corrections. When elements are large, 95\%-errors are below $10 \%$ with $\widetilde{\Lambda}$, when the beam 657 number is greater than 100.

658 The expectation of the radiuses of the confidence interval $E 95_{\widetilde{\Lambda}}$ and $E 95_{\widetilde{\Lambda}}^{n_{v}}$ (green dashed line 659 in Figure 9 and 10) are very close to the expectation of the $95 \%$ error, showing again that the 660 estimated confidence intervals are consistent.
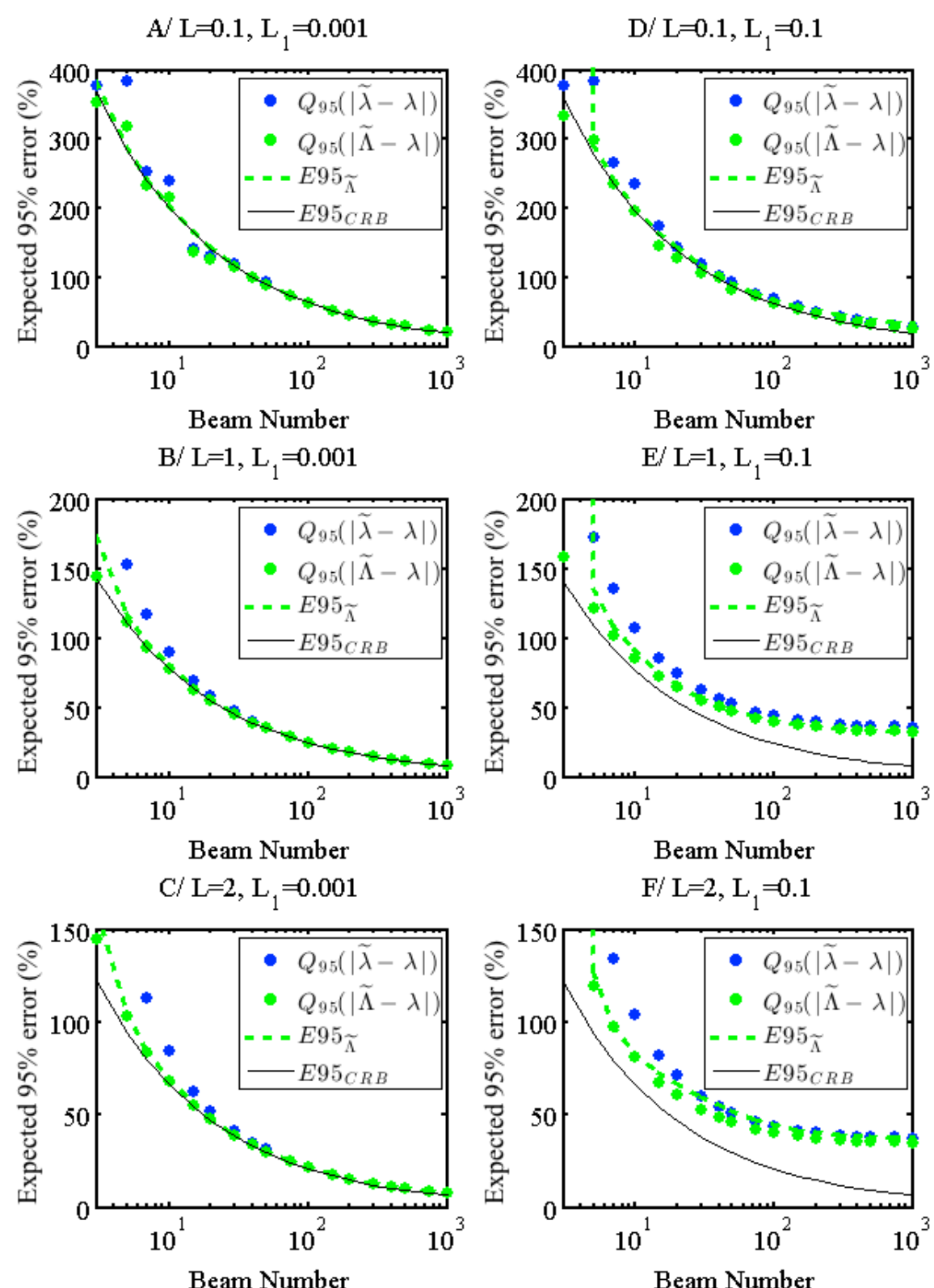

$\mathrm{F} / \mathrm{L}=2, \mathrm{~L}_{1}=0.1$

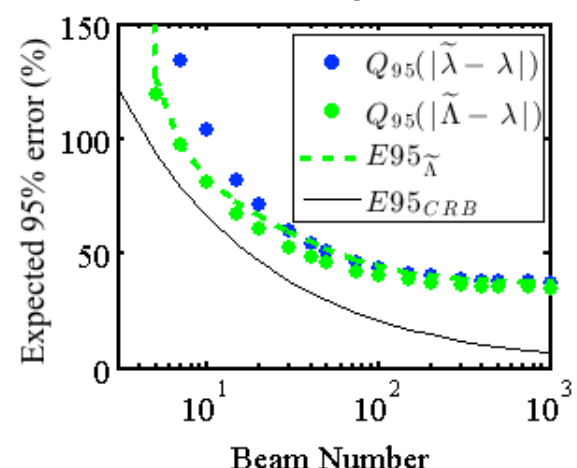

661 
662 Fig. 9. Expectations of the $95 \%$ error, expressed in percentage of $\lambda$, as the $95^{\text {th }}$ percentile of the

663 absolute residual to $\lambda$ for $\tilde{\lambda}$ (blue dots) and $\tilde{\Lambda}$ (green dots). We show for comparison the estimated 664 radius of the confidence interval $E 95_{\widetilde{\Lambda}}$ (green dashed line) and the radius bound derived from the 665 Cramer Rao bound $E 95_{\mathrm{CRB}}$ (black line).

666
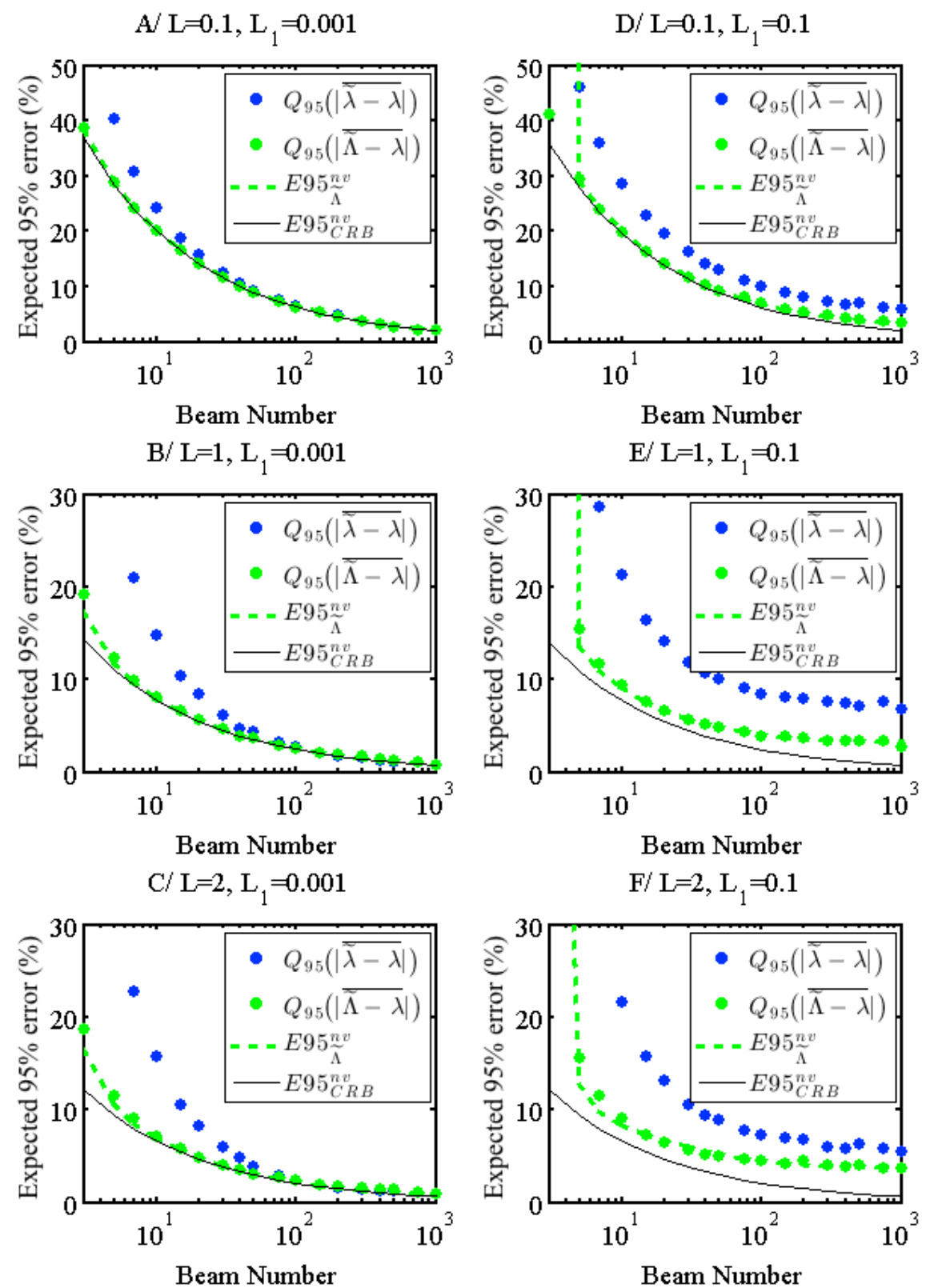

667

668 Fig. 10. Same as Fig. 9 for an average over 100 voxels. Expectations of the $95 \%$ error over nv=100

669 voxels, expressed in percentage of $\lambda$, as the $95^{\text {th }}$ percentile of the absolute averaged residual to $\lambda$ for $\tilde{\lambda}$

670 (blue dots) and $\widetilde{\Lambda}$ (green dots). We show for comparison the estimated radius of the confidence 
671 interval $E 95_{\widetilde{\Lambda}}^{n v}$ (green dashed line) and the radius bound derived from the Cramer Rao bound $E 95_{\mathrm{CRB}}^{n v}$ 672 (black line).

673

674 Figures 11 and 12 show similar trends to Figures 9 and 10, for both biased and unbiased Beer675 Lambert estimators. When blue dots are missing (usual Beer-Lambert), they correspond to 676 cases where $\mathrm{I}=1$ in more than $5 \%$ of the voxel, so that the $95 \%$ error is in this case infinite. As 677 expected from previous results, the estimator of the radius of the $95 \%$ confident interval 678 (green dashed lines) is not consistent for the average over 100 voxels, in ranges where when $\widehat{\Lambda}$ 679 is biased (typically $\mathrm{L}_{\text {and }} \mathrm{L}_{1}$ large, Fig. 12F). 

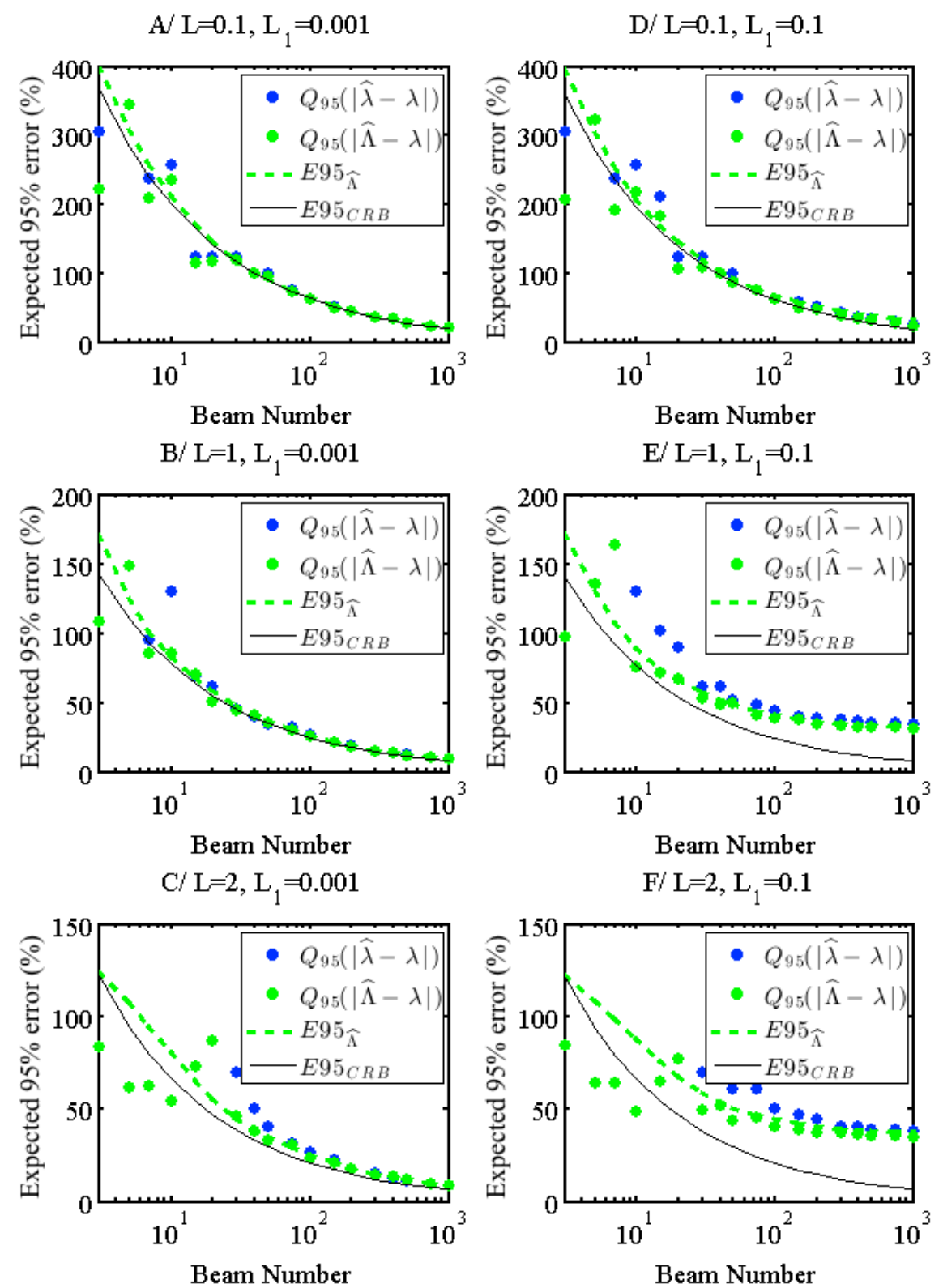

680

681 Fig. 11. Same as Fig. 9 for the Beer-Lambert estimators. Expectations of the 95\% error, expressed in

682 percentage of $\lambda$, as the $95^{\text {th }}$ percentile of the absolute residual to $\lambda$ for $\hat{\lambda}$ (blue dots) and $\widehat{\Lambda}$ (green dots).

683 We show for comparison the estimated radius of the confidence interval $E 95_{\widehat{\Lambda}}$ (green dashed line) and 684 the radius bound derived from the Cramer Rao bound $E 95_{\mathrm{CRB}}$ (black line). 

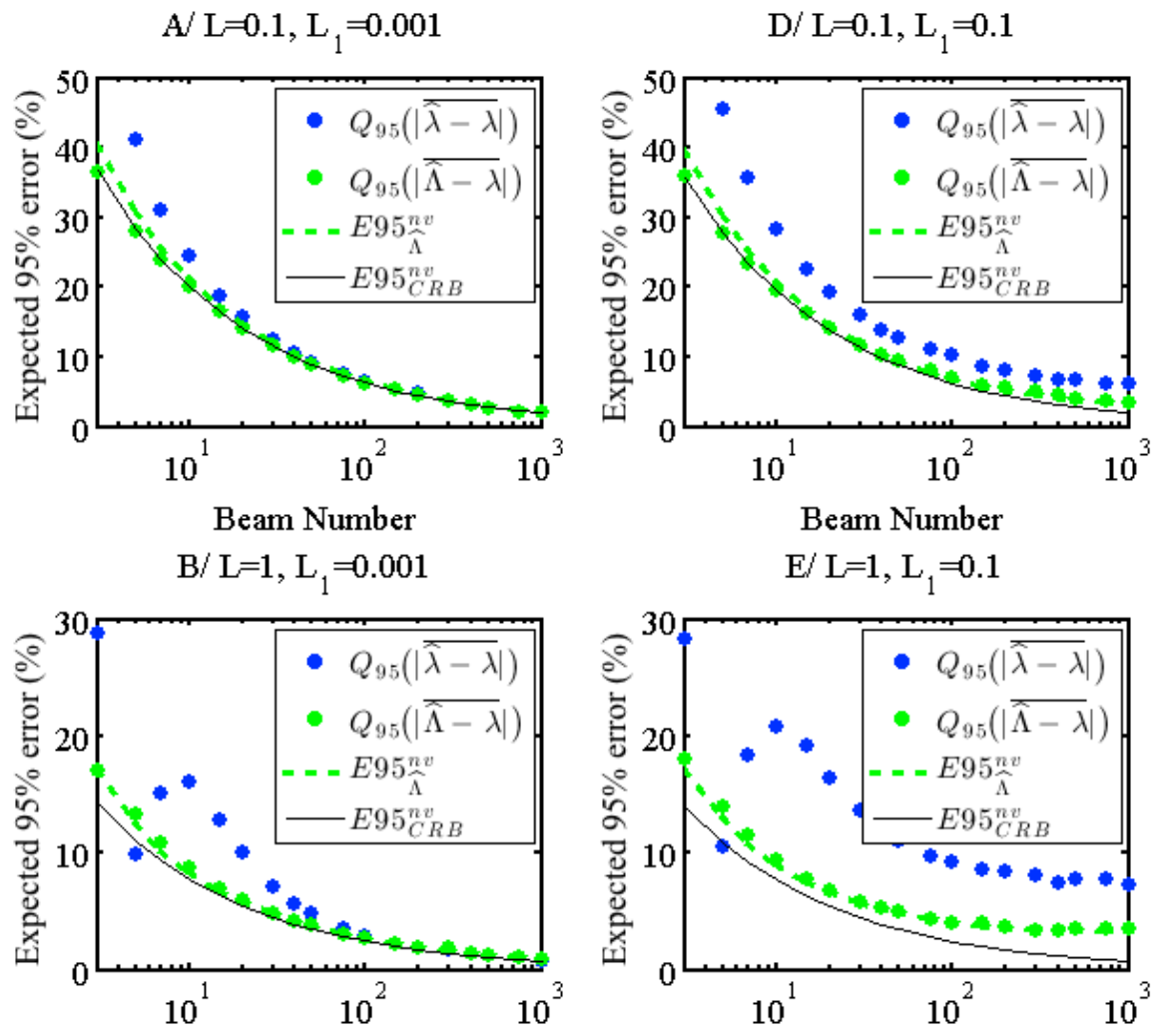

$\mathrm{E} / \mathrm{L}=1, \mathrm{~L}_{1}=0.1$

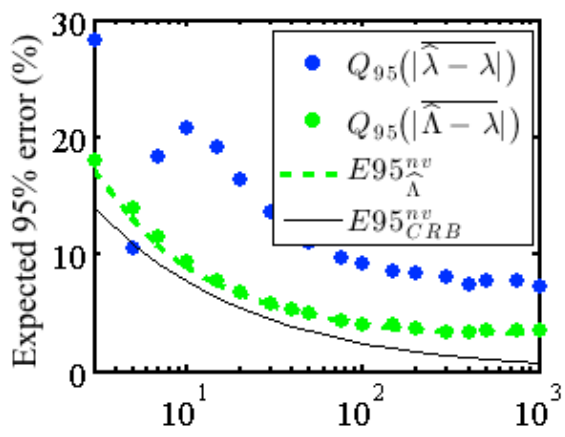

Beam Number

$\mathrm{C} / \mathrm{L}=2, \mathrm{~L}_{1}=0.001$

Beam Number

$\mathrm{F} / \mathrm{L}=2, \mathrm{~L}_{1}=0.1$
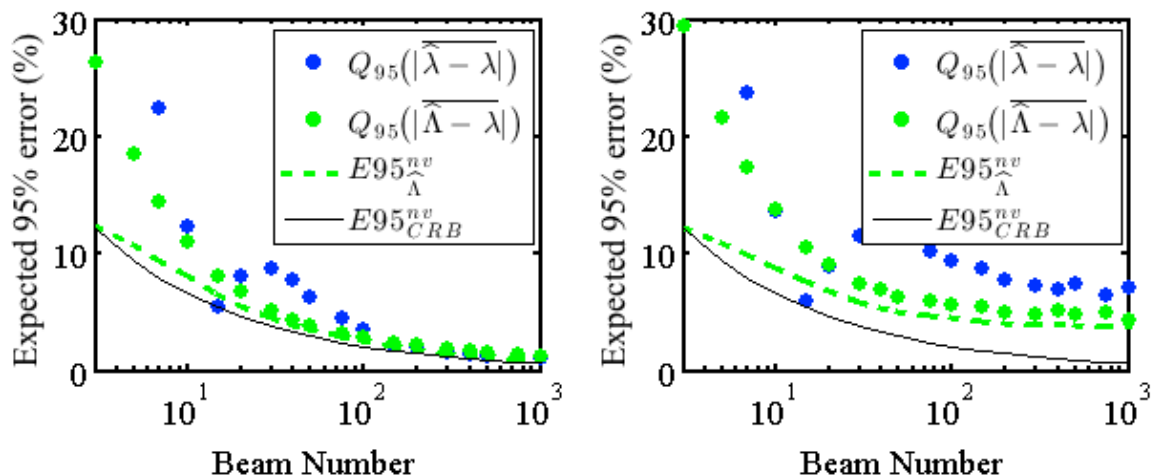

688 Fig. 12. Same as Fig. 10 for the Beer-Lambert estimators. Expectations of the $95 \%$ error over nv=100

689 voxels, expressed in percentage of $\lambda$, as the $95^{\text {th }}$ percentile of the absolute averaged residual to $\lambda$ for $\hat{\lambda}$

690 (blue dots) and $\widehat{\Lambda}$ (green dots). We show for comparison the estimated radius of the confidence

691 interval $E 95_{\widehat{\Lambda}}^{n v}$ (green dashed line) and the radius bound derived from the Cramer Rao bound $E 95_{\mathrm{CRB}}^{n v}$

692 (black line).

693 
696

697

698 In this subsection, we show the statistics of estimators computed with simulations described

\subsection{Estimator performance for unequal path lengths}

699 in subsection 4.3, in the context of a spherical voxel (unequal path lengths). Since MLE performance is similar to the results shown in the previous section, it is not shown again.

Here, we focus on the comparison between Beer-Lambert estimators $\hat{\lambda}$ and $\widehat{\Lambda}_{2}$ (similar to $\widehat{\Lambda}$,

but which includes the correction for unequal path lengths). This is of major importance, since

703

704

705

706

707

708

709

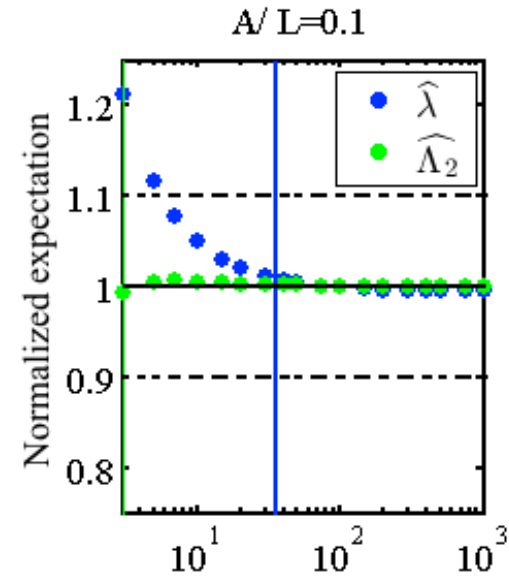

Beam Number

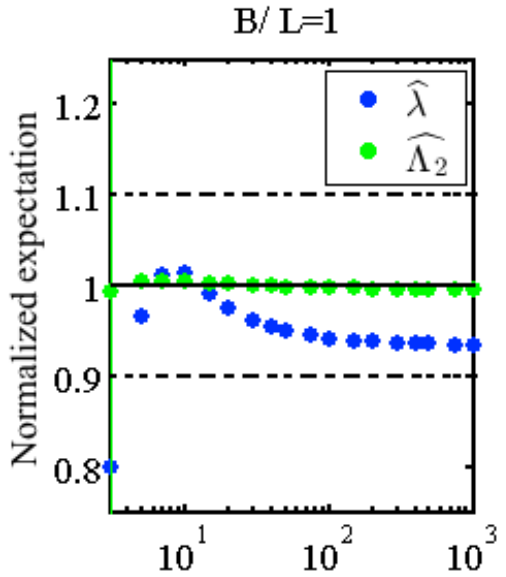

Beam Number

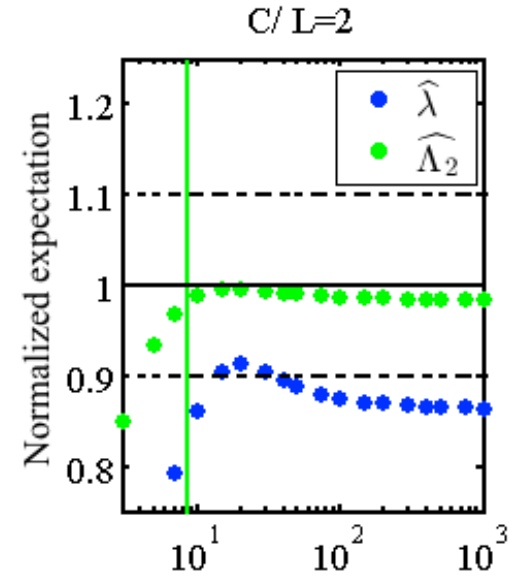

Beam Number

Fig. 13. Same as Figure 4 for the unequal path lengths. Expectations of the Beer-Lambert-attenuationcoefficient estimators, as a function of the beam number. Blue dots corresponds the usual Beer-

714 Lambert estimator $(\hat{\lambda})$. Green dots corresponds to the unbiased Beer-Lambert estimator $\left(\widehat{\Lambda}_{2}\right)$, that 
accounts for unequal path length, element size and beam number and extended definition when $\mathrm{I}=1$.

716 Estimators are normalized by their true value $\lambda$, so that they are consistent when the expectation equals

717 to one. The vertical lines correspond to the lowest values of $\mathrm{N}$ leading to a bias smaller than $1 \%$ in

718 blue and green, for respectively the biased and unbiased estimators.

\subsubsection{Estimator efficiency}

721 Figure 14 show the variances of $\widehat{\lambda}$ and $\widehat{\Lambda}_{2}$ similarly to Fig. 6. For low density (Fig. 14A), the

722 variances of $\hat{\lambda}$ and $\widehat{\Lambda}_{2}$ are similar to those obtained with equal path lengths (Fig. 6A). When 723 density is higher (Fig. $14 \mathrm{~B}$ and C), the variance of $\widehat{\Lambda}_{2}$ (Green dots) is much larger, which is mostly explained by the variability of the empirical path lengths. $\sigma_{\widehat{\Lambda}_{2}}^{2}$ (green dashed line) provides consistent estimators for the variance of $\widehat{\Lambda}_{2}$, at least when the beam number is larger than 10. This variance, however, is significantly larger than the Cramer-Rao bound (black line), showing that $\widehat{\Lambda}_{2}$ is suboptimal when $N$ is smaller than 100 , contrary to the MLE, that reaches the Cramer-Rao bound (not shown). Again, $\hat{\lambda}$ being biased, its variance cannot be assessed against the Cramer-Rao bound to evaluate its efficiency.

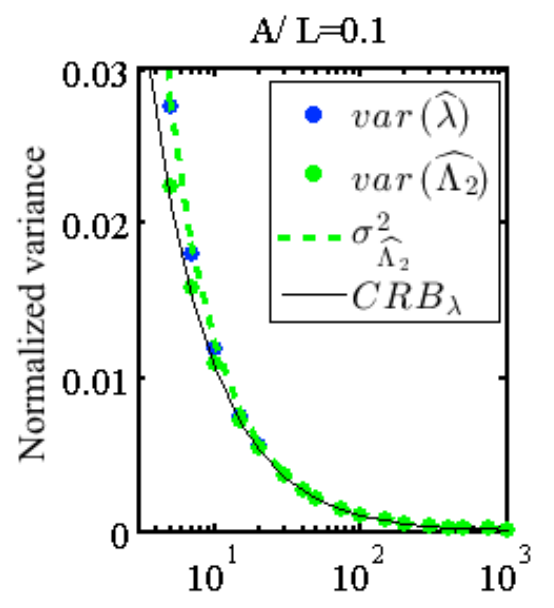

Beam Number

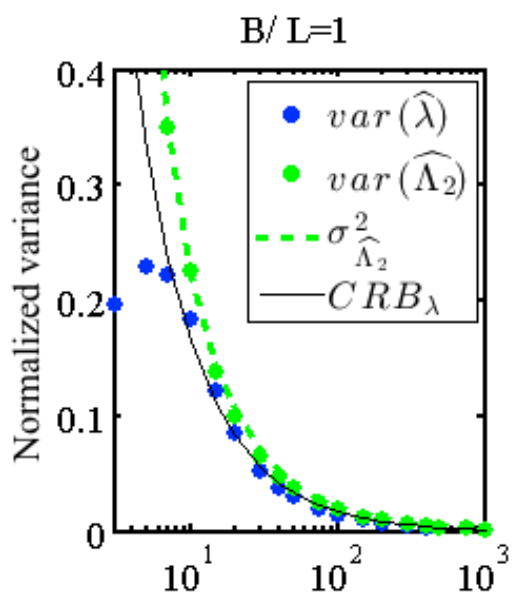

Beam Number

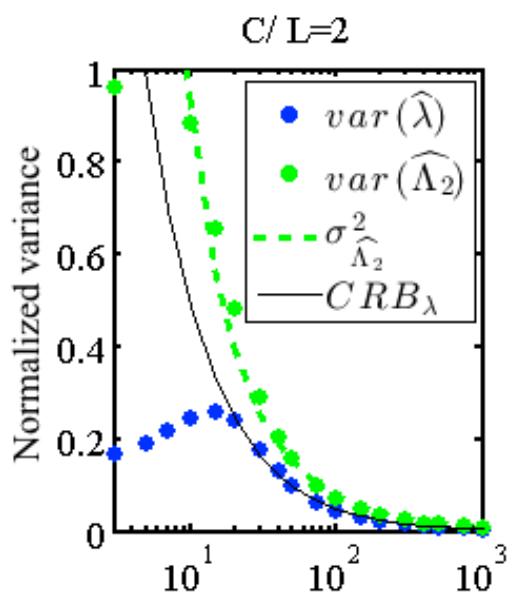

Beam Number

731 Fig. 14. Same as Figure 6 for unequal path lengths. Normalized variances of Beer-Lambert attenuation

732 coefficient estimators, as a function of the beam number. Normalized variances correspond to variances multiplied by $\delta^{2}$. Blue dots corresponds to the variance of the usual Beer-Lambert estimator 
$734 \hat{\lambda}$. Green dots corresponds to the variance of the unbiased Beer-Lambert estimator $\widehat{\Lambda}_{2}$. Green dashed

735 lines correspond to the dimensionless expectation of the variance estimator $\sigma_{\widehat{\Lambda}_{2}}^{2}$. The black line 736 corresponds to Cramer-Rao bound for the variance of unbiased estimator.

\subsubsection{5\% error of estimators for a single and a group of voxels}

739 The coverage probabilities of the estimated confidence interval $\widehat{\Lambda}_{2} \pm z_{\alpha / 2} \sqrt{\sigma_{\widehat{\Lambda}_{2}}^{2}}$ are similar to

740 those shown for $\widehat{\Lambda} \pm z_{\alpha / 2} \sqrt{\sigma_{\widehat{\Lambda}}^{2}}$ in Figure 8 (and thus not shown). More interestingly, Figures 15

741 and 16 show the expectation of the 95\% error, as in Figure 11 and 12. The 95\% errors are

742 significantly reduced at the scale of a single voxel when the density is high and $\mathrm{N}$ is large

743 (Fig. 15C). As for the other bias correction, the error reduction is limited in other cases since

744 estimators are too uncertain. When averaged at the scale of several voxels, the benefit of the

745 correction for unequal path lengths is clearly visible when the beam number is moderate and

746 large (Fig. 16B and C). In these cases, 95\% errors are always greater than 7 and 12\%, even

747 when the beam number is large. Bias correction leads to an important reduction of the error,

748 that becomes close to the Cramer-Rao bound. However, contrary to unbiased MLE for which

749 the Cramer Rao bound is reached with unequal path length (not shown here, but logical since

750 the formulation is not affected by the path length), the Beer-Lambert estimator is not optimal

751 even after bias corrections. This is especially true when the beam number is small, because of

752 the variability induced by the empirical correction factor $a_{e}$. This demonstrates that the

753 unbiased MLE is clearly more efficient than the (unbiased) Beer-Lambert estimator, since

754 those estimators are mostly computed in cubic voxels. 
756

757

758

759

760

761

762

763

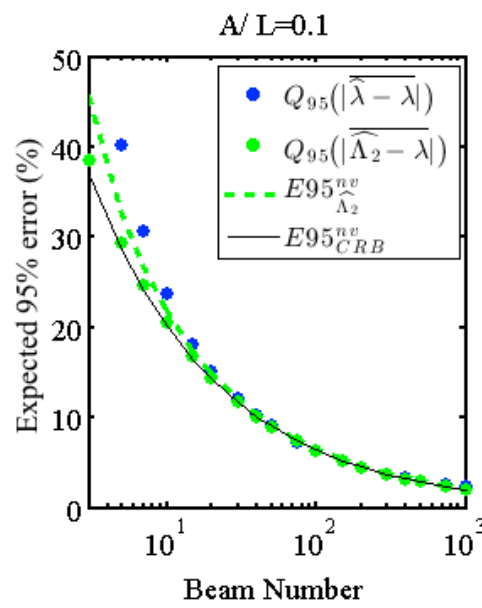

$\mathrm{A} / \mathrm{L}=0.1$

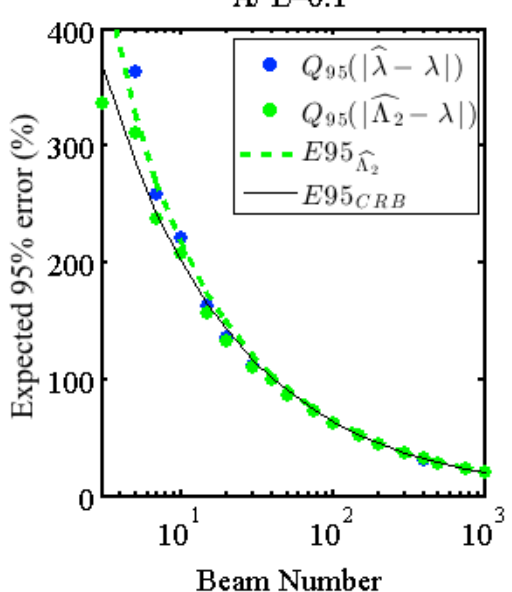

Beam Number
$\mathrm{B} / \mathrm{L}=1$

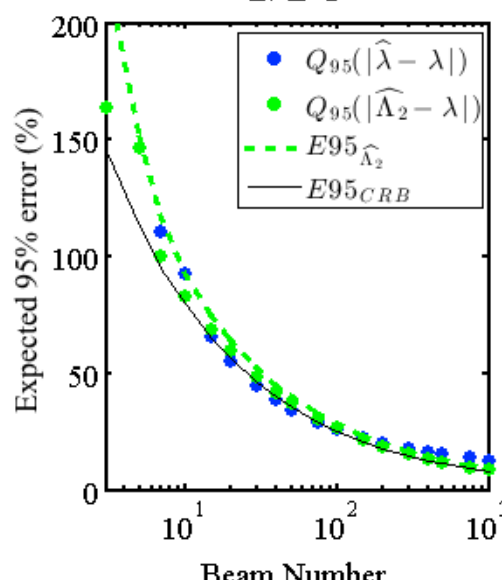

Beam Number

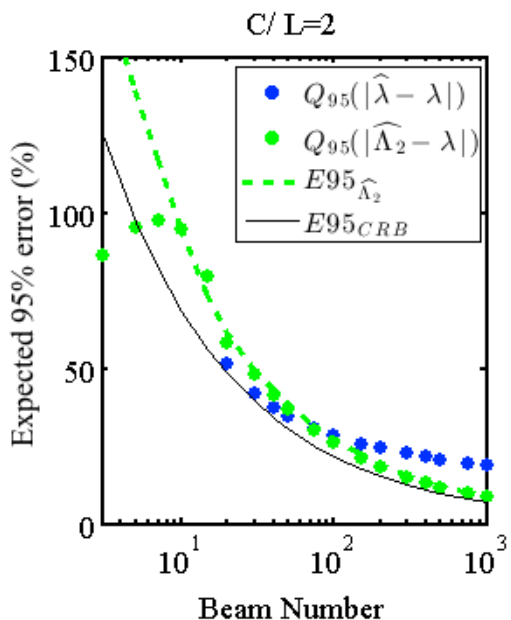

Beam Number

Fig. 15. Same as Fig. 11 for the unequal path lengths. Expectations of the $95 \%$ error, expressed in percentage of $\lambda$, as the $95^{\text {th }}$ percentile of the absolute residual to $\lambda$ for $\hat{\lambda}$ (blue dots) and $\widehat{\Lambda}_{2}$ (green dots). We show for comparison the estimated radius of the confidence interval $E 95_{\widehat{\Lambda}_{2}}$ (green dashed line) and the radius bound derived from the Cramer Rao bound $E 95_{\mathrm{CRB}}$ (black line).

764 Fig. 16. Same as Fig. 12 for unequal path length. Expectations of the $95 \%$ error over nv=100 voxels,
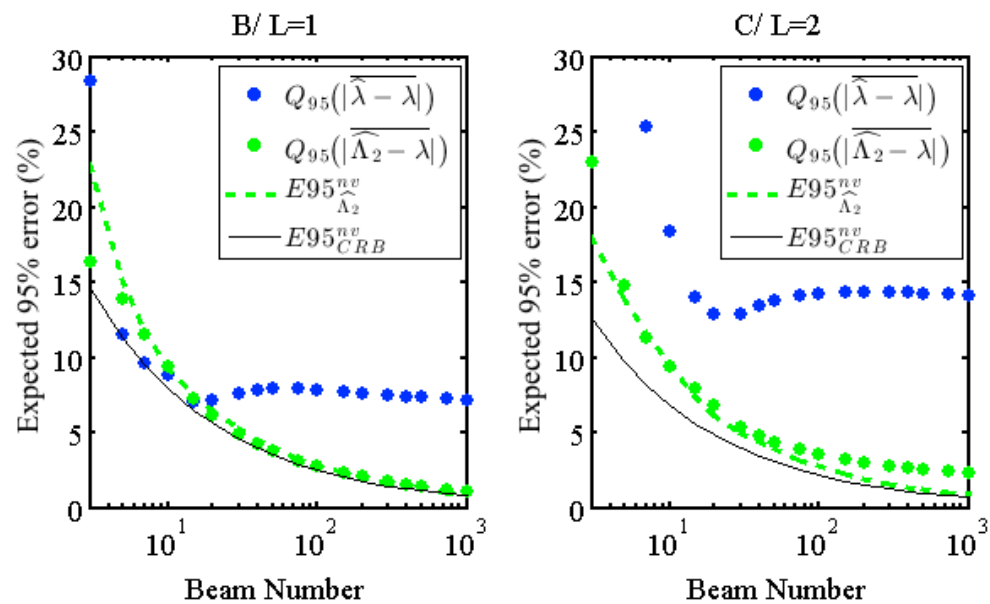
expressed in percentage of $\lambda$, as the $95^{\text {th }}$ percentile of the absolute averaged residual to $\lambda$ for $\hat{\lambda}$ (blue dots) and $\widehat{\Lambda}_{2}$ (green dots). We show for comparison the estimated radius of the confidence interval $E 95_{\widehat{\Lambda}_{2}}^{n v}$ (green dashed line) and the radius bound derived from the Cramer Rao bound $E 95_{\mathrm{CRB}}^{n v}$ (black line). 
770

771

772 
773

774

775

776

777

778

779

780

781

782

783

784

785

786

787

788

789

790

791

792

793

794

795

796

797

\section{Discussion}

The method proposed here is based on a mathematical formulation of the estimation problem in a formal statistical framework. Technical derivations are detailed in several supplementary materials for reference, to facilitate the reading of the manuscript. This theoretical part is completed with numerical simulations, for validation and determination of the range of validity of our new estimators. As every modeling approach, both mathematical formulation and simulations are based on assumptions that are not necessarily valid in the field. Here, we assume that the spatial distribution of beams is random, whereas the actual one has a periodic pattern, potentially altered by occlusion. Also, we assume simple square leaves for our vegetation elements. More realistic patterns for beam shooting and vegetation elements can be simulated (Grau et al., 2017). However, a drawback of this later approach is that it limits both theoretical derivations and simulation number. The benefits of our simplifying assumptions are that the mathematical framework can be deeply explored and that the cost of numerical simulations is very limited, so that a full statistical analysis of estimator performance can be done, over a wide range of parameter values (here element size, voxel size and beam number).

Our derivations entails to propose some new ready-to-use, analytical estimators for the attenuation coefficient, which is proportional to PAD/LAD. These estimators generalize the ones proposed in several pioneering studies. In this sense, our unbiased Beer-Lambert estimator combines the effects of finite-element size and unequal path lengths that are already identified respectively in Béland et al. (2014a), and Béland et al. (2014b) and Grau et al. (2017). Regarding the effect of the element size, we choose to explicitly correct our estimator for the associated bias, rather than to restrict its range of validity to largest voxels, as in Béland et al. (2014a). For unequal path lengths, our formulation is more general than the 
798

799

800

801

802

803

804

805

806

807

808

809

810

811

812

813

814

815

816

817

818

819

820

821

822

empirical correction proposed in Béland et al. (2014b), since it does not assume a particular shape for the voxel. Contrary to the secant method, used for example in Bailey and Mahafee (2017b), our formulation is analytical and easy to implement. Also, our Beer-Lambert estimators are defined even when the RDI is equal to one, whereas earlier formulation considered this special case as "occluded" (Béland et al., 2014a), leading to a negative bias as shown above. Our approach also demonstrates that the modified contact frequency, introduced in Béland et al. (2011), is indeed the Maximum Likelihood Estimator of the mathematical problem. It extends the modified contact frequency initially developed for infinitely small elements to the case of finite-size elements in a theoretically-based formulation. This formulation slightly differs from the one proposed in the discussion in Béland et al. (2014a), in which the correction term that accounts for finite-element size is the same as for the Beer-Lambert law (see Eq. (12)). Such a proposition is not supported by the Maximum Likelihood, but numerical consequences are probably limited. More importantly, our formulation includes some bias corrections that depend on the beam number, for both approaches (Beer-Lambert and MLE), whereas usual estimators are shown to be positively biased to more than $20 \%$ when the beam number is small. To the best of our knowledge, such an effect has never been reported before.

Numerical simulations show that the new estimators are consistent for a much wider range of parameter values (element size, attenuation coefficient, beam number), than the usual ones. The range of consistency of the unbiased MLE $\widetilde{\Lambda}$ is wider than the one of the unbiased BeerLambert estimator. Interestingly, the beam number required to reach consistency of the unbiased MLE depends on the element depth $L_{1}$ only. Contrary to the unbiased Beer-Lambert estimator, for which a larger number of beams is required when the vegetation density increases, the unbiased MLE is not affected by the actual value of the attenuation coefficient. 
823 This is practically convenient, since the attenuation coefficient is unknown prior to the 824 computation of the estimates when dealing with field data. When $L_{1} \leq 0.1$, which is the case

825 for most vegetation when the voxel size is larger than $5 \mathrm{~cm}, \widetilde{\Lambda}$ is consistent (bias smaller than $8261 \%$ ) as soon as the beam number is larger than 5. This is important when computing the 827 attenuation in small voxels, because the beam number in often low (e.g. Béland et al., 2009), 828 especially when vegetation is dense because of occlusion. Another potential application is airborne LiDAR, for which the point density is much lower than TLS. For comparison, more

830 than 100 beams are required in vegetation with $L_{1} \leq 0.01$ and $L \leq 2$ to reach the same 831 consistency with the usual Beer-Lambert estimator as with the unbiased MLE. Another benefit of the MLE when compared to the Beer-Lambert estimator is that it does not require any bias correction when path lengths are unequal. This is all the more important, that the bias correction for unequal path lengths tends to reduce the efficiency of this estimator (in comparison with Cramer-Rao bound). We also demonstrate that the unbiased MLE is

836 efficient, at least when the element size is small, since it reaches the Cramer-Rao bound. This means that no unbiased estimator can have a smaller error than this estimator, so that the unbiased MLE can be considered as optimal. This result is of major importance, since it shows that there is no need for further correction, as long as the assumptions leading to these results are valid.

842 Our mathematical derivations allow us to derive estimators of the variance and hence, confidence interval for the unbiased estimators. Analyzing their coverage properties shows

844 that they are generally consistent, especially when using the formulation based on both the 845 "Agresti-Coull" interval and the basic interval, depending whether the voxel density is low or 846 high (Eq. 46). Providing such confidence intervals fill a gap for voxel-based approach, as 847 done earlier for gap fraction methods in Zhao et al. (2015). The first outcome of our 
848 confidence interval estimates is that the prediction at voxel scale is uncertain, especially when

849 the voxels are small with errors larger than $100 \%$, since the beam number is low and the 850 variability of the vegetation sample is high. When elements are large, uncertainty remains

851 high, even when scanning density is very high, because of the variability of element positions

852 within vegetation samples. The confidence intervals of the mean attenuation coefficient in a

853 larger volume (eventually discretized in small voxels) is much narrower, since the total beam 854 number is larger and the impact of the variability of vegetation samples is dampened. Our 855 numerical results, however, might be affected by our assumptions (random position, square 856 flat leaves, random beams), so that it would be worthwhile in the future to evaluate the 857 asymptotic residual variability in the context of realistic vegetation, for example following the 858 approach detailed in Grau et al. (2017).

860 Until now, most of the evaluation of the performance of voxel-based estimators was based on 861 the analysis of residual error between estimations and field data. The different sources of bias 862 and dispersion were thus merged. We believe that the applications of the new estimators and 863 their confidence interval should help to choose the appropriate resolution. Small voxels lead 864 to a smaller probability to get larger gaps that invalidate the assumption of random 865 distribution and results in an underestimation of LAD (Béland et al., 2014a). In other words, 866 transmission laws are wider-than-exponential in presence of spatial correlations (Davis and 867 Marshak, 2004; Pimont et al., 2009; Larsen and Clark, 2014). The question of resolution is 868 critical, since the recommendations vary among studies from some millimeters to $2 \mathrm{~m}$ (e.g. 869 Hosoi and Asama, 2006; Grau et al. 2017; Béland et al., 2011; Béland et al., 2014a; Pimont et 870 al., 2015; Bailey and Mahafee, 2017b). Among others, these studies deal with various 871 vegetation (various element size from needles to large leaves, various spatial distribution, 872 single tree vs forestry plot, etc.) as well as various scanning density (from single scan to high 
873 density scanning). Also, the formulations vary among studies, some biases being corrected in

874 some studies, while not corrected in others. Most of them are affected by the positive biases

875 caused by the beam number and the element size (with the exception of Béland et al., 2014a

876 in which element size sensitivity is evaluated). Such biases are stronger at high resolution

877 since the beam number is lower and the element path is larger. Some of them are affected by

878 the negative biases of the usual Beer-Lambert estimator when path lengths are unequal, or

879 when the RDI is equal to 1 . Again, such biases both vary with voxel resolution and

880 orientation. A general use of the unbiased MLE $\widetilde{\Lambda}$, for instance, should cancel these biases and

881 thus gives the opportunity to focus on the remaining sources of bias and dispersion pointed

882 out in the introduction. Among them, we can cite the TLS "flaws" (partial hit and detection

883 threshold) and the remaining vegetation "flaws" (element orientation and clumping, leaf and

884 wood separation). Vegetation heterogeneity is especially concerned by the issue of the voxel

885 size. At the end, the computation of confidence intervals could also help determine the

886 resolution that minimizes errors, since the resolution that minimizes the confidence interval

887 radius of the average attenuation coefficient within a given volume could be selected.

888

889

\section{Conclusion}

890

891 The present work provides an innovative approach of TLS point clouds, based on both

892 analytical derivations and numerical simulations to propose some new efficient estimators of 893 the attenuation coefficient, which is proportional to the LAD/PAD. These estimators are

894 designed for TLS point clouds of high density, so that they mostly concern TLS, although

895 their consistency with low beam number is also promising for their application to airborne

896 scanner. Among them, the unbiased MLE is consistent and efficient in a wider range of

897 parameter values than the usual estimators. It accounts for statistical biases associated with 
898 beam number and element size. Although the biases caused by partial hit and clumping at 899 larger scale are not included, this new estimator should improve the choice of voxel

900 resolution, since it corrects several biases that depends on resolution and that might have been

901 mixed up in some earlier studies. Also, this work provides some estimators for the confidence

902 intervals of the attenuation coefficient within a volume containing one or several voxels,

903 increasing our knowledge of $\mathrm{PAD} / \mathrm{LAD}$ regarding measurement accuracy by TLS, which is

904 probably lower than expected when voxels are small, and again contributes to the 905 determination of the best resolution.

906

907

908 Funding: The PACA region and INRA funded the PhD of one of the co-authors.

909 
910

911

912

913

914 Bailey, B.N., Mahafee, W.F., 2017a. Rapid, high-resolution measurement of leaf area and leaf

915 orientation using terrestrial LiDAR scanning data. Meas. Sci. Technol. 28(6).

916

917

918

919

920

921

922

923

924

925

926

927

928

929

930

931

932

933

934

\section{References}

Allard, D., Lopez-Lozano, R., Baret, F., 2013 Modeling forest canopies with a hierarchical multi-ring Boolean model for estimating Leaf Area Index. Spat. Stat-Neth 5, 42-56

Bailey, B.N., Mahafee, W.F., 2017b. Rapid measurement of the three-dimensional distribution of leaf orientation and the leaf angle probability density function using terrestrial LiDAR scanning. Remote Sens. Environ. 194, 63-76.

Béland, M., Widlowski, J.-L., Fournier, R.A., Côté, J.-F., Verstraete, M., 2011. Estimating leaf area distribution in savanna trees from terrestrial LiDAR measurements. Agric. For. Meteorol. 151, 1252-1266.

Béland, M., Baldocchi, D.D., Widlowski, J.-L., Fournier, R.A., Verstraete, M.M., 2014a. On seing the wood from the leaves and the role of voxel size in determining leaf area distribution of forests with terrestrial LiDAR. Agric. For. Meteorol. 184, 82-97.

Béland, M., Widlowski, J.-L., Fournier, R.A., 2014b. A model for deriving voxel-level tree leaf area density estimates from ground-based LiDAR. Environ. Model. Softw. 51, 184-189.

Brown, L.D., Cai T.T., DasGupta, A., 2001. Interval Estimation for a Binomial Proportion. Stat. Sci. 16(2), 101-133. 
935 Chen, J.M., Cihlar, J., 1995. Quantifying the effect of canopy architecture on optical

936 measurements of leaf area index using two gap size analysis methods. Geosci. Remote Sens.

937 IEEE Trans. 33, 777-787.

938

939 Campbell G.S. \& Norman J.N. (1998) An Introduction to Environmental Biophysics 2nd 940 ed. Springer, New York.

941

942 Davis, A.B., Marshak, A., 2004. Photon propagation in heterogeneous optical media with

943 spatial correlations: enhanced mean-free-paths and wider-than-exponential free-path 944 distributions. J. Quant. Spectrosc. Ra. 84, 3-34.

945

946 Durrieu, S., Allouis, T., Fournier, R.A., Véga, C., Albrech, L., 2008. Spatial quantification of 947 vegetation density from terrestrial laser scanner data for characterization of 3D Forest 948 structure at plot level. In Proceedings of the SilviLaser, Edinburgh, UK, 17-19 September 9492008.

950

951 Grau., E., Durrieu, S., Fournier, R., Gastellu-Etchegorry, J.P., Yin, T., 2017. Estimation of 3D 952 vegetation density with Terrestrial Laser Scanning data using voxels. A sensitivity analysis of 953 influencing parameters. Remote Sens. Environ. 191, 373 - 388.

954

955 Hebert, M., Kroktov, E., 1992. 3-D Measurements from imaging laser radars: How good are 956 they? Int. J. Image Vis. Comput. 10, 170-178.

957 
958 Hosoi, F., Omasa, K., 2006. Voxel based 3D modeling of individual trees for estimating leaf

959 area density using high-resolution portable scanning LiDAR. IEEE Trans. Geosci. Remote 960 Sens. 44, 3610-3618.

961

962 Hosoi, F., Omasa, K., 2007. Factors contributing to accuracy in the estimation of the woody 963 canopy leaf area density profile using 3D portable LiDAR imaging. J. Exp. Bot. 58, 34639643473.

965

966 Jupp, D.L.B., Culvenor, D.S., Lovell, J.L., Newnham, G.J., Strahler, H., and Woodcock, C.E., 967 2009. Estimating forest LAI profiles and structural parameters using a ground- based laser 968 called "Echidna”. Tree Physiol. 29, 171-181.

969

970 Kay, S.M., 1993. Fundamentals of Statistical Signal Processing: Estimation Theory, Prentice $971 \quad$ Hall, 595 p.

972

973 Keane, R.E., 2015. Wildland fuel fundamentals and application. Springer International 974 Publishing, Switzerland, $191 \mathrm{p}$.

975

976 Kukenbrink, D., Schneider, F.D., Leiterer, R., Schaepman M.E., Morsdorf F., 2017. 977 Quantification of hidden canopy volume of airborne laser scanning data using a voxel 978 traversal algorithm. Remote Sens. Environ. 194, 424-436.

979

980 Nilson, T., 1971. A theoretical analysis of the frequency of gaps in plant stands. Agric.

981 Meteorol. 8 (C), 25-38. 
983 Norman, J.M., Campbell, G.S., 1989. Canopy structure. In: Pearcy, R.W., Ehleringer, 984 J.,Mooney, H.A., Rundel, P.W. (Eds.), Plant Physiological Ecology - Field Methodsand 985 Instrumentation. Chapman and Hall, London, pp. 301-325.

986

987 Larsen, M.L., Clark, A.S., 2014. On the link between particle size and deviations from the 988 Beer-Lambert-Bouguer law for direct transmission. J. Quant. Spectrosc. Ra. 133, 646-651.

989

990 Pimont. F., Dupuy, J.-L., Caraglio, Y., Morvan, D., 2009. Effect of vegetation heterogeneity 991 on radiative transfer in forest fires. Int. J. Wildland Fire 18, 536-553.

992

993 Pimont. F., Dupuy, J.-L., Rigolot, R., Prat, V., Piboule, A., 2015. Estimating Leaf Bulk 994 Density Distribution in a Tree Canopy Using Terrestrial LiDAR and a Straightforward 995 Calibration Procedure. Remote Sens. 7(6), 7995-8018.

996

997 Pisek, J., Sonnentag, O., Richardson, A.D., ottus, M.M., 2013. Is the spherical leaf inclination 998 angle distribution a valid assumption for temperate and boreal broadleaf tree species? Agric. 999 For. Meteorol. 169, 186-194.

1000

1001 Ross, J., 1981. The Radiation Regime and Architecture of Plant Stands. Task for Vegetation 1002 Sciences 3; Springer: The Hague, The Netherlands, p. 391.

1003

1004 Ruel, J.J., Ayres, M.P., 1999. Jensen's inequality predicts effects of environmental variation. 1005 Trends Ecol. Evol., 14, 361-366.

1006 
1007 Schneider, R., Weil, W., 2008. Stochastic and integral geometry. Springer Science \& 1008 Business Media.

1009

1010 Seielstad, C., Stonesifer, C., Rowell, E., Queen, L., 2011. Deriving Fuel Mass by Size Class 1011 in Douglas-fir (Pseudotsuga menziesii) Using Terrestrial Laser Scanning. Remote Sens. 3, 1012 1691-1709.

1013

1014 Stoyan, D., W. Kendall, J. Mecke. 1987. Stochastic Geometry and Its Applications. J. Wiley 1015 \& Sons/Akademie-Verlag, Berlin 1987, 345 pp.

1016

1017 Vierling, K.T., Vierling, L.A., Gould, W.A., Martinuzzi, S., Clawges, R.M., 2008. Lidar: 1018 shedding new light on habitat characterization and modeling. Frontiers Ecol. Environ. 6, 90101998.

1020

1021 Warren Wilson J., 1960. Inclined point quadrats. New Phytol. 59(1), 1-8.

1022

1023 Zhao, F., Yang, X., Schull, M.A., Román-Colón, M.O., Yao, T., Wang, Z., Zhang, Q., Jupp, 1024 D.L., Lovell, J.L., Culvenor, D.S., 2011. Measuring effective leaf area index, foliage profile: 1025 and stand height in New England forest stands using a full-waveform ground-based lidar. 1026 Remote Sens. Environ. 115, 2954-2964.

1027

1028 Zhao, K., García, M., Liu, S., Guo, Q., Chen, G., Zhang, X., Zhou, Y., Meng, X., 2015.

1029 Terrestrial lidar remote sensing of forests: Maximum likelihood estimates of canopy profile, 1030 leaf area index, and leaf angle distribution. Agric. For. Meteorol. 209-201, 100-113. 
1032

Supplementary Material

1033

Supplementary S1. Expected range of the optical depth of an element of vegetation in a

1034 voxel

1035 Following the definition of $\lambda_{1}$ in Eq. (9), the optical depth of an element in a cubic voxel of size $\delta$ is

$$
\mathrm{L}_{1}=\lambda_{1} \delta=\frac{\mathrm{S}_{1}}{\mathrm{~S}} \approx \frac{\mathrm{S}_{1}}{\delta^{2}}
$$

1036 For a needle of radius $r$ and length 1 , this leads to:

$$
\lambda_{1} \delta \approx \frac{2 \pi r l}{4 \delta^{2}}
$$

1037 For a (small) needle of diameter $2 \mathrm{r}=0.5 \mathrm{~mm}$ and length $1=5 \mathrm{~cm}$, we have:

$$
\lambda_{1} \delta_{\min } \approx 210^{-5} \delta^{-2}
$$

1038 For a flat leaf of radius $r$, this leads to:

$$
\lambda_{1} \delta \approx \frac{2 \pi r^{2}}{4 \delta^{2}}
$$

1039 For a (large) leaf of diameter $2 \mathrm{r}=10 \mathrm{~cm}$, we have:

$$
\lambda_{1} \delta_{\text {max }} \approx 510^{-3} \delta^{-2}
$$


1043

1044

1045

1046

1047

1048

1049

1050

1051

1052

1053

1054

1055

1056

1057

1058

1059

1060

1061

\section{Supplementary S2. Point estimators and their variance based on Beer-Lambert law}

The usual Beer-Lambert estimator is based on the RDI. It assumes that (i) a mean path length $\bar{\delta}(\sim \mathrm{V} / \mathrm{S})$ is suitable to account for unequal path length in the voxel and (ii) $-\log (1-\mathrm{I})$ is a good estimator $\hat{\kappa}$ of $\kappa=$ $-\log (1-E(I))$ (Note that $\kappa$ is the optical depth of the voxel). However, in both cases, the non-linearity of the $\log$ function limits the validity of such assumptions and makes the standard estimator $\hat{\lambda}$ a biased estimator of $\lambda$.

Taking the $\log (1-x)$ of Eq. (20), we have:

$$
\log (1-E(\mathrm{I}))=\log \left(\frac{1}{\mathrm{~S}} \iint_{\mathrm{S} \in \mathrm{S}}\left(1-\lambda_{1} \delta(\mathrm{s})\right)^{\lambda / \lambda_{1}} \mathrm{dS}\right)
$$

The Lemma proved below enables to approximate the logarithm in (S2-1) and gives a second order approximation of $\log (1-E(\mathrm{I}))$, as a function of the actual attenuation coefficient $\lambda$. Combining (S2-1) with the Lemma leads to:

$$
\log (1-E(\mathrm{I})) \approx-\overline{\delta_{e}} \lambda+\frac{1}{2} \sigma_{\delta_{e}}^{2} \lambda^{2}
$$

where the effective mean path length $\overline{\delta_{e}}$ and its variance $\sigma_{\delta_{e}}^{2}$ are defined as the mean and variance of the effective path lengths $\delta_{e, j}=-\frac{\log \left(1-\lambda_{1} \delta_{j}\right)}{\lambda_{1}}$.

We then consider the issue of the bias associated with $\log (1-\mathrm{I})$. A bias correction can be computed applying the approximation S6-5 to the function $\mathrm{g}(\mathrm{x})=\log (1-\mathrm{x})$, which depends on $\mathrm{g}$ "(I) and the variance of $\mathrm{I}$ (given by Eq. 23). An unbiased estimator of $\hat{\kappa}$ is thus

$$
\hat{\kappa}=-\widehat{\log (1}-\mathrm{E}(\mathrm{I}))=-\log (1-\mathrm{I})-\frac{1}{2}\left(\frac{\mathrm{I}(1-\mathrm{I})}{\mathrm{N}}+\sigma_{\mathrm{I}_{\infty}}^{2}\right)(1-\mathrm{I})^{-2}
$$

Combining (S2-2) and (S2-3) leads to a second order polynomial in $\lambda$ that can be solved to derive the corrected estimator $\widehat{\Lambda}_{2}$ accounting for unequal path lengths (S2-7).

$$
\log (1-\mathrm{I})-\frac{1}{2}\left(\frac{\mathrm{I}(1-\mathrm{I})}{\mathrm{N}}+\sigma_{\mathrm{I}_{\infty}}^{2}\right)(1-\mathrm{I})^{-2}=-\overline{\delta_{e}} \lambda+\frac{1}{2} \sigma_{\delta_{e}}^{2} \lambda^{2}
$$

We first derive the estimator for nearly equal path length $\widehat{\Lambda}$. When path lengths are nearly constant $\left(\sigma_{\delta_{e}}^{2} \approx 0\right)$, the equation S2-4 in $\lambda$ leads to:

$$
\widehat{\Lambda}=-\frac{1}{\overline{\delta_{e}}}\left(\log (1-\mathrm{I})+\frac{\mathrm{I}}{2 \mathrm{~N}(1-\mathrm{I})}+\frac{\sigma_{\mathrm{I}_{\infty}}^{2}}{2(1-\mathrm{I})^{2}}\right) \approx-\frac{1}{\overline{\delta_{e}}}\left(\log (1-\mathrm{I})+\frac{\mathrm{I}}{2 \mathrm{~N}(1-\mathrm{I})}\right)
$$


1062 Numerical simulations show that $\frac{\sigma_{\mathrm{I}_{\infty}}^{2}}{2(1-\mathrm{I})^{2}}$ is always very small compared to $\log (1-\mathrm{I})$ in the range of interest. It is

1063 thus neglected in the rest of the study.

1064

1065 For unequal path length, the second order polynomial in $\lambda$ of (S2-4) can be rewritten (since by definition of $\widehat{\Lambda}$,

$\left.1066 \log (1-E(\mathrm{I}))=-\overline{\delta_{e}} \widehat{\Lambda}\right)$

$$
\frac{1}{2} \sigma_{\delta_{e}}^{2} \lambda^{2}-\overline{\delta_{e}} \lambda+\overline{\delta_{e}} \widehat{\Lambda}=0
$$

1067 Assuming that $2 \frac{\sigma_{\delta_{e}}^{2}}{\delta_{e}} \widehat{\Lambda}$, is smaller than 1 , we can solve the polynomial and keep the smallest root. This leads to

1068 the $\widehat{\Lambda}_{2}$ estimator that accounts for unequal path lengths:

$$
\widehat{\Lambda}_{2}=\frac{\overline{\delta_{e}}}{\sigma_{\delta_{e}}^{2}}\left(1-\sqrt{1-2 \frac{\sigma_{\delta_{e}}^{2}}{\overline{\delta_{e}}} \widehat{\Lambda}}\right)
$$

1069 The above indices are not defined when $\mathrm{I}=1$, since the Beer-Lambert approach does not provide any insight 1070 regarding the attenuation coefficient rather than "probably high". As explained in section 3.2, the center of the confidence interval can be estimated as a function of $\mathrm{N}$, by the Agresti-Coull interval. With $z_{\alpha / 2}^{2}=1$, it is:

$$
\mathrm{I}_{c}=\frac{1+\frac{1}{2 \mathrm{~N}}}{1+\frac{1}{\mathrm{~N}}}=1-\frac{\frac{1}{2 \mathrm{~N}}}{1+\frac{1}{\mathrm{~N}}}=1-\frac{1}{2 N+2}
$$

1072 Since $\mathrm{I}_{\mathrm{c}}$ is at the center of the confidence interval, $-\frac{\log \left(1-\mathrm{I}_{c}\right)}{\bar{\delta}_{e}}$ is a more robust estimator for $\lambda$ in this context:

$$
\widehat{\Lambda}=\frac{\log (2 \mathrm{~N}+2)}{\overline{\delta_{e}}}
$$

1073 The estimator of the variance of $\widehat{\Lambda}$ is derived from $(\mathrm{S} 6-2)$. Let $g(x)=-\left(\log (1-\mathrm{x})+\frac{\mathrm{x}}{2 \mathrm{~N}(1-\mathrm{x})}\right)$

$$
g^{\prime}(x)=\frac{1}{1-x}-\frac{(1-x)-x(-1)}{2 N(1-x)^{2}}=\frac{1}{1-x}\left(1-\frac{1}{2 N(1-x)}\right)
$$

1074 We can thus define the estimator of variance of $\widehat{\Lambda}$ as:

$$
\sigma_{\widehat{\Lambda}}^{2}=\frac{\sigma_{I}^{2}}{\delta_{e}(1-\mathrm{I})^{2}}\left(1-\frac{1}{2 \mathrm{~N}(1-\mathrm{E}(\mathrm{I}))}\right)^{2}
$$

1075 Estimating the variance of $\widehat{\Lambda}_{2}$ as defined in (S2-7) can be done using (S6-2):

$$
\sigma_{\widehat{\Lambda}_{2}}^{2}=\sigma_{\widehat{\Lambda}}^{2}\left(\frac{\overline{\delta_{e}}}{\sigma_{\delta_{e}}^{2}} \frac{\frac{1}{2} 2 \frac{\sigma_{\delta_{e}}^{2}}{\overline{\delta_{e}}}}{\sqrt{1-2 \frac{\sigma_{\delta_{e}}^{2}}{\overline{\delta_{e}}}}}\right)^{2}=\frac{\sigma_{\widehat{\Lambda}_{2}}^{2}}{1-2 \frac{\sigma_{\delta_{e}}^{2}}{\overline{\delta_{e}}} \widehat{\Lambda}} \approx \sigma_{\widehat{\Lambda}_{2}}^{2}\left(1+2 \frac{\sigma_{\delta_{e}}^{2}}{\overline{\delta_{e}}} \widehat{\Lambda}+4\left(\frac{\sigma_{\delta_{e}}^{2}}{\overline{\delta_{e}}} \widehat{\Lambda}\right)^{2}\right)
$$


1079 With $\mathrm{g}(\mathrm{x})=\log (x)$, the integral formulation of (S6-1) leads to:

$$
\frac{1}{S} \iint_{s \in S} \log \left(\left(1-\lambda_{1} \delta(s)\right)^{\lambda / \lambda_{1}}\right) d S \approx \log \left(\frac{1}{S} \iint_{s \in S}\left(1-\lambda_{1} \delta(s)\right)^{\frac{\lambda}{\lambda_{1}}} \mathrm{~d} S\right)-\frac{1}{2} \frac{\operatorname{var}\left(\left(1-\lambda_{1} \delta(s)\right)^{\lambda / \lambda_{1}}\right)}{\frac{\left(1-\lambda_{1} \delta(s)\right)^{\lambda / \lambda_{1}}}{{ }^{2}}}
$$

1080 The left member is:

$$
\frac{1}{\mathrm{~S}} \iint_{\mathrm{s} \in \mathrm{S}} \log \left(\left(1-\lambda_{1} \delta(\mathrm{s})\right)^{\lambda / \lambda_{1}}\right) \mathrm{d} S=\frac{\lambda}{\lambda_{1}} \overline{\log \left(1-\lambda_{1} \delta\right)}=-\overline{\delta_{e}} \lambda
$$

$1081 \quad$ With $\mathrm{g}(\mathrm{x})=\exp (\lambda x), \operatorname{var}\left(\left(1-\lambda_{1} \delta(\mathrm{s})\right)^{\lambda / \lambda_{1}}\right)=\operatorname{var}\left(g\left(\frac{\log \left(1-\lambda_{1} \delta(\mathrm{s})\right)}{\lambda_{1}}\right)\right)$

1082 According to (S6-2),

$$
\begin{gathered}
\operatorname{var}\left(\left(1-\lambda_{1} \delta(s)\right)^{\lambda / \lambda_{1}}\right) \approx g^{\prime}\left(\overline{\frac{\log \left(1-\lambda_{1} \delta(s)\right)}{\lambda_{1}}}\right)^{2} \operatorname{var}\left(\frac{\log \left(1-\lambda_{1} \delta(s)\right)}{\lambda_{1}}\right) \\
=\lambda^{2} \exp \left(\lambda \frac{\overline{\log \left(1-\lambda_{1} \delta(s)\right)}}{\lambda_{1}}\right)^{2} \operatorname{var}\left(\delta_{e}\right)
\end{gathered}
$$

1083 Since at the first order, $g(\bar{x})=\overline{g(x)}$,

1084 we can write, $\exp \left(\lambda \overline{\frac{\log \left(1-\lambda_{1} \delta(\mathrm{s})\right)}{\lambda_{1}}}\right) \approx \overline{\exp \left(\lambda \frac{\log \left(1-\lambda_{1} \delta(\mathrm{s})\right)}{\lambda_{1}}\right)}=\overline{\left(1-\lambda_{1} \delta(\mathrm{s})\right)^{\lambda / \lambda_{1}}}$, and then:

$$
\frac{1}{2} \frac{\operatorname{var}\left(\left(1-\lambda_{1} \delta(\mathrm{s})\right)^{\lambda / \lambda_{1}}\right)}{{\overline{\left(1-\lambda_{1} \delta(s)\right)^{\lambda / \lambda_{1}}}}_{2}^{2}} \approx \frac{1}{2} \lambda^{2} \operatorname{var}\left(\delta_{e}\right)
$$

1085 Combining this with the above results leads to:

$$
\log \left(\frac{1}{\mathrm{~S}} \iint_{\mathrm{s} \in \mathrm{S}}\left(1-\lambda_{1} \delta(\mathrm{s})\right)^{\frac{\lambda}{\lambda_{1}}} \mathrm{dS}\right) \approx-\overline{\delta_{e}} \lambda+\frac{1}{2} \sigma_{\delta_{e}}^{2} \lambda^{2}
$$


1087

1088

1089

1090

$$
\mathcal{L}\left(\lambda ; \mathrm{z}_{1}, \mathrm{z}, \ldots, \mathrm{z}_{\mathrm{N}}\right)=\prod_{j=1}^{N} \mathrm{f}_{Z}\left(\mathrm{z}_{\mathrm{j}} ; \delta_{\mathrm{j}}\right)=\prod_{\mathrm{z}_{\mathrm{j}}<\delta_{\mathrm{j}}} \lambda\left(1-\lambda_{1} \mathrm{z}_{\mathrm{j}}\right)^{\lambda / \lambda_{1}-1} \prod_{\mathrm{z}_{\mathrm{j}}=\delta_{\mathrm{j}}}\left(1-\lambda_{1} \mathrm{z}_{\mathrm{j}}\right)^{\lambda / \lambda_{1}}
$$

\section{Supplementary S3. Point and variance estimators based on MLE}

\section{S3.1. Log likelihood and MLE of the attenuation coefficient}

Let use denote $\left\{\mathrm{z}_{j}\right\}_{j=1, \mathrm{~N}}$ the $\mathrm{N}$ free paths with respective path lengths $\left\{\delta_{j}\right\}_{j=1, \mathrm{~N}}$.

From Eq. (17), the likelihood of $\mathrm{Z}$ is:

1093

The ML estimator is the value $\tilde{\lambda}$ that cancels the first derivative of $\mathcal{L}$ (Kay, 1993, chapter 7). Deriving the

1092 logarithm of the likelihood and equating to zero provides

$$
\frac{\mathrm{d} \log \mathcal{L}}{\mathrm{d} \lambda}=\frac{\mathrm{N}_{\mathrm{i}}}{\lambda}+\sum_{\mathrm{j}=1}^{\mathrm{N}} \frac{\log \left(1-\lambda_{1} \mathrm{z}_{\mathrm{j}}\right)}{\lambda_{1}}=0
$$

1094

\section{S3.2. Bias correction terms for the MLE}

1096

The bias correction for the MLE is derived from (S6-6) with $\mathrm{f}(x, y)=\frac{\mathrm{x}}{\mathrm{y}}$, since the MLE is $\frac{\mathrm{I}}{\bar{z}_{e}}$. The three terms

1097 corresponding to bias corrections are, assuming that beams are independent:

$$
-\frac{1}{2} \sigma_{I}^{2} \frac{\partial^{2} f}{\partial x^{2}}\left(I, \overline{z_{e}}\right)=-\frac{1}{2} \sigma_{I}^{2} \times 0=0
$$

1098

$$
-\frac{1}{2} \sigma_{\overline{z_{e}}}^{2} \frac{\partial^{2} f}{\partial y^{2}}\left(I, \overline{z_{e}}\right)=-\frac{1}{2} \sigma_{\overline{z_{e}}}^{2} \frac{2 \mathrm{I}}{\overline{z_{e}}{ }^{3}}=-\frac{\mathrm{I}}{N \bar{z}_{e}{ }^{3}} \operatorname{var}\left(z_{e}\right)
$$

1099

$$
-\sigma_{I, \overline{z_{e}}} \frac{\partial^{2} f}{\partial x \partial y}\left(I, \overline{z_{e}}\right)=\sigma_{I, \overline{z_{e}}} \frac{1}{{\overline{z_{e}}}^{2}}=\frac{1}{N{\overline{z_{e}}}^{2}} \operatorname{covar}\left(\mathbf{1}_{\mathrm{z}_{\mathrm{j}}<\delta_{\mathrm{j}}}, z_{e}\right)
$$

1100 Combining S3-4, S3-5, and S3-6 leads to:

$$
\tilde{\Lambda}=\frac{\mathrm{I}}{\overline{z_{e}}}-\frac{\mathrm{I}}{N{\overline{z_{e}}}^{3}} \operatorname{var}\left(z_{e}\right)+\frac{1}{N{\overline{z_{e}}}^{2}} \operatorname{covar}\left(\mathbf{1}_{\mathrm{z}_{\mathrm{j}}<\delta_{\mathrm{j}}}, z_{e}\right)
$$

1101 Although practically computable for a distribution of $\left\{\mathrm{z}_{\mathrm{j}}\right\}$ and $\left\{\delta_{\mathrm{j}}\right\}$, the variance of the estimator can not be 1102 analytically derived, so that it is not possible to estimate the variance and thus the confidence interval for the 
1103 estimation. The next subsection is dedicated to the development of analytical estimator for $\operatorname{var}\left(z_{e}\right)$ and

$1104 \operatorname{covar}\left(\mathbf{1}_{\mathrm{z}_{\mathrm{j}}<\delta_{\mathrm{j}}}, z_{e}\right)$

1105

1106 S3.3. Estimates for $\operatorname{var}\left(z_{e}\right)$ and covar $\left(\mathbf{1}_{\mathrm{z}_{\mathrm{j}}<\delta_{\mathrm{j}}}, z_{e}\right)$

1107 From variance formulae:

$$
\operatorname{var}\left(z_{e}\right)=E\left[z_{e}{ }^{2}\right]-E\left[z_{e}\right]^{2}
$$

1108 From the probability distribution (Eq. (17)) and definition of $z_{e}$, the expectation of $z_{e}$ is:

$$
E\left[z_{e}\right]=\frac{1}{S} \int_{S}\left(\int_{0}^{\delta(s)}-\frac{\log \left(1-\lambda_{1} z\right)}{\lambda_{1}} \lambda\left(1-\lambda_{1} \mathrm{z}\right)^{\lambda / \lambda_{1}-1} d z+-\frac{\log \left(1-\lambda_{1} \delta(s)\right)}{\lambda_{1}}\left(1-\lambda_{1} \delta(s)\right)^{\lambda / \lambda_{1}}\right) d s
$$

1109 Integrating by parts the integral leads to:

$$
\begin{aligned}
E\left[z_{e}\right]=\frac{1}{S} \int_{S}\left(\left[\frac{\log \left(1-\lambda_{1} \mathrm{z}\right)}{\lambda_{1}}\left(1-\lambda_{1} \mathrm{z}\right)^{\frac{\lambda}{\lambda_{1}}}\right]_{0}^{\delta(\mathrm{s})}\right. \\
\left.\quad-\int_{0}^{\delta(\mathrm{s})}-\frac{1}{1-\lambda_{1} \mathrm{z}}\left(1-\lambda_{1} \mathrm{z}\right)^{\frac{\lambda}{\lambda_{1}}} d z-\frac{\log \left(1-\lambda_{1} \delta(\mathrm{s})\right)}{\lambda_{1}}\left(1-\lambda_{1} \delta(\mathrm{s})\right)^{\frac{\lambda}{\lambda_{1}}}\right) d s \\
=\frac{1}{S} \int_{S}\left(\int_{0}^{\delta(\mathrm{s})}\left(1-\lambda_{1} \mathrm{z}\right)^{\frac{\lambda}{\lambda_{1}}-1} d z\right) d s=\frac{P(Z<\delta)}{\lambda}=\frac{E\left[\mathbf{1}_{\mathrm{z}_{\mathrm{j}}<\delta_{\mathrm{j}}}\right]}{\lambda}
\end{aligned}
$$

1110 This demonstrates that:

$$
\lambda=\frac{E\left[\mathbf{1}_{\mathrm{z}_{\mathrm{j}}<\delta_{\mathrm{j}}}\right]}{E\left[z_{e}\right]}=\frac{I_{\infty}}{{\overline{z_{e}}}^{\infty}}
$$

1111 Similarly,

$$
E\left[z_{e}{ }^{2}\right]=\frac{1}{S} \int_{S}\left(\int_{0}^{\delta(s)}\left(\frac{\log \left(1-\lambda_{1} \mathrm{z}\right)}{\lambda_{1}}\right)^{2} \lambda\left(1-\lambda_{1} \mathrm{z}\right)^{\lambda / \lambda_{1}-1} d z+\left(\frac{\log \left(1-\lambda_{1} \delta(\mathrm{s})\right)}{\lambda_{1}}\right)^{2}\left(1-\lambda_{1} \delta(\mathrm{s})\right)^{\lambda / \lambda_{1}}\right) d s
$$

1112 Integrating twice by parts the integral leads to: 


$$
\begin{aligned}
E\left[z_{e}{ }^{2}\right]=\frac{1}{S} \int_{S}\left(\left[-\left(\frac{\log \left(1-\lambda_{1} \mathrm{z}\right)}{\lambda_{1}}\right)^{2}\left(1-\lambda_{1} \mathrm{z}\right)^{\frac{\lambda}{\lambda_{1}}}\right]_{0}^{\delta(\mathrm{s})}\right. \\
\quad-\int_{0}^{\delta(\mathrm{s})} \frac{2}{1-\lambda_{1} \mathrm{z}}\left(\frac{\log \left(1-\lambda_{1} \mathrm{z}\right)}{\lambda_{1}}\right)\left(1-\lambda_{1} \mathrm{z}\right)^{\frac{\lambda}{\lambda_{1}}} d z+\left(\frac{\log \left(1-\lambda_{1} \delta(\mathrm{s})\right)}{\lambda_{1}}\right)^{2}(1 \\
\left.\left.-\lambda_{1} \delta(\mathrm{s})\right)^{\frac{\lambda}{\lambda_{1}}}\right) d s=\frac{2}{\lambda} \frac{1}{\mathrm{~S}} \int_{S}\left(\int_{0}^{\delta(\mathrm{s})}-\frac{\log \left(1-\lambda_{1} \mathrm{z}\right)}{\lambda_{1}} \lambda\left(1-\lambda_{1} \mathrm{z}\right)^{\frac{\lambda}{\lambda_{1}}-1} d z\right) d s \\
=\frac{2}{\lambda} E\left[\mathbf{1}_{\mathrm{z}_{\mathrm{j}}<\delta_{\mathrm{j}}} \mathrm{z}_{e}\right]
\end{aligned}
$$

Thus, $\quad \operatorname{var}\left(z_{e}\right)=\frac{2}{\lambda} E\left[\mathbf{1}_{\mathrm{z}_{\mathrm{j}}<\delta_{\mathrm{j}}} \mathrm{z}_{e}\right]-E\left[z_{e}\right]^{2}$

And $\operatorname{covar}\left(\mathbf{1}_{\mathrm{z}_{\mathrm{j}}<\delta_{\mathrm{j}}}, z_{e}\right)=E\left[\mathbf{1}_{\mathrm{z}_{\mathrm{j}}<\delta_{\mathrm{j}}} z_{e}\right]-E\left[\mathbf{1}_{\mathrm{z}_{\mathrm{j}}<\delta_{\mathrm{j}}}\right] E\left[z_{e}\right]$

1114 S3.4. Point estimator

1115 Plugging S3-14 and S3-15 in, S3-7 leads to:

$$
\widetilde{\Lambda}=\frac{\mathrm{I}}{\bar{z}_{e}}-\frac{1}{N} \frac{\mathrm{I}}{\bar{z}_{e}{ }^{3}}\left(\frac{2}{\lambda} E\left[\mathbf{1}_{\mathrm{z}_{\mathrm{j}}<\delta_{\mathrm{j}}} \mathrm{z}_{e}\right]-E\left[z_{e}\right]^{2}\right)+\frac{1}{N \bar{z}_{e}{ }^{2}}\left(E\left[\mathbf{1}_{\mathrm{z}_{\mathrm{j}}<\delta_{\mathrm{j}}} z_{e}\right]-E\left[\mathbf{1}_{\mathrm{z}_{\mathrm{j}}<\delta_{\mathrm{j}}}\right] E\left[z_{e}\right]\right)
$$

1116 And since $E\left[\mathbf{1}_{\mathrm{z}_{\mathrm{j}}<\delta_{\mathrm{j}}}\right] \approx I, E\left[z_{e}\right] \approx \overline{z_{e}}, E\left[\mathbf{1}_{\mathrm{z}_{\mathrm{j}}<\delta_{\mathrm{j}}} z_{e}\right] \approx \overline{\mathbf{1}_{\mathrm{z}_{\mathrm{j}}<\delta_{\mathrm{j}}} z_{e}}$ and $\lambda \approx \frac{\mathrm{I}}{\overline{z_{e}}}$ :

$$
\widetilde{\Lambda}=\frac{\mathrm{I}}{\overline{z_{e}}}-\frac{\overline{\mathbf{1}_{\mathrm{z}_{\mathrm{j}}<\delta_{\mathrm{j}}} Z_{e}}}{N{\overline{z_{e}}}^{2}}
$$

1117

1118 S3.5. Variance estimator

1119 The variance for the MLE is derived from (S6-4) with $\mathrm{f}(x, y)=\frac{\mathrm{x}}{\mathrm{y}}$, since the MLE is $\frac{\mathrm{I}}{\overline{z_{e}}}$. The three terms

1120 corresponding to bias corrections are:

$$
\sigma_{I}^{2}\left(\frac{\partial f}{\partial x}\right)^{2}\left(I, \overline{z_{e}}\right)=\frac{\sigma_{I}^{2}}{{\overline{z_{e}}}^{2}}=\frac{I(1-I)}{N{\overline{z_{e}}}^{2}}=\frac{I}{N{\overline{z_{e}}}^{2}}-\frac{I^{2}}{N{\overline{z_{e}}}^{2}}
$$

1121

$$
\sigma_{\overline{z_{e}}}^{2}\left(\frac{\partial f}{\partial y}\right)^{2}\left(I, \overline{z_{e}}\right)=\frac{\operatorname{var}\left(z_{e}\right)}{N} \frac{\mathrm{I}^{2}}{{\overline{z_{e}}}^{4}}=\frac{1}{N}\left(\frac{2}{\lambda} \overline{\mathbf{1}_{\mathrm{z}_{\mathrm{J}}<\delta_{\mathrm{J}}} z_{e}}-{\overline{z_{e}}}^{2}\right) \frac{\mathrm{I}^{2}}{{\overline{z_{e}}}^{4}}=\frac{2}{\lambda} \frac{\mathrm{I}^{2}}{N{\overline{z_{e}}}^{4}} \overline{\mathbf{1}_{\mathrm{z}_{\mathrm{J}}}<\delta_{\mathrm{J}} Z_{e}}-\frac{\mathrm{I}^{2}}{N{\overline{z_{e}}}^{2}}
$$




$$
\begin{gathered}
2 \sigma_{I, \overline{z_{e}}}\left(\frac{\partial f}{\partial x}\right)\left(I, \overline{z_{e}}\right)\left(\frac{\partial f}{\partial y}\right)\left(I, \overline{z_{e}}\right)=2 \frac{\left(\overline{\mathbf{1}_{\mathrm{z}_{\mathrm{j}}<\delta_{\mathrm{j}}} \bar{z}_{e}}-I \overline{z_{e}}\right)}{N}\left(\frac{1}{\overline{\bar{z}_{e}}}\right)\left(-\frac{\mathrm{I}}{\overline{\bar{z}_{e}}}\right) \\
=-\overline{\mathbf{1}_{\mathrm{z}_{\mathrm{j}}}<\delta_{\mathrm{j}} \bar{z}_{e}} \frac{2 I}{N \overline{\bar{z}_{e}}}+\frac{2 I^{2}}{N{\overline{z_{e}}}^{2}}
\end{gathered}
$$

1123 Since $\lambda \approx \frac{\mathrm{I}}{\overline{z_{e}}}$, summing S3-18 to S3-20 leads to:

$$
\operatorname{var}\left(\frac{I}{\overline{z_{e}}}\right) \approx \frac{I}{N \bar{z}_{e}^{2}}
$$

1124 (S3-14) can be rewritten:

$$
\tilde{\Lambda}=\frac{\mathrm{I}}{\overline{z_{e}}}\left(1-\frac{\overline{\mathbf{1}_{\mathrm{z}_{\mathrm{j}}<\delta_{\mathrm{j}}} \bar{z}_{e}}}{N I \overline{z_{e}}}\right)=\operatorname{MLE}_{\lambda}\left(1-\frac{\overline{\mathbf{1}_{\mathrm{j}}<\delta_{\mathrm{j}} z_{e}}}{N I \overline{z_{e}}}\right)
$$

$1125 \mathrm{MLE}_{\lambda}$ is corrected by a factor of which the variance is assumed to be small when compared to the variance of the 1126 standard MLE, so that, we can write (when I $>0$ ):

$$
\sigma_{\tilde{\Lambda}}^{2}=\frac{I}{N{\overline{z_{e}}}^{2}}\left(1-\frac{\overline{\mathbf{1}_{\mathrm{z}_{1}<\delta_{1}} \bar{z}_{e}}}{N I \overline{z_{e}}}\right)^{2}
$$

1127 This formulation does not account for the asymptotic variability of attenuation coefficient estimators, caused by

1128 the variability of element positions in vegetation samples. However, this asymptotic variability can be estimated 1129 from Eq. (31):

$$
\lim _{N \rightarrow \infty} \sigma_{\widehat{\Lambda}}^{2}=\frac{\sigma_{\mathrm{I}_{\infty}}^{2}\left(\mathrm{I}, \lambda_{1} \bar{\delta}\right)}{{\overline{\delta_{e}}}^{2}(1-I)^{2}}
$$

1130 And thus:

$$
\sigma_{\tilde{\Lambda}}^{2}=\frac{I}{N{\overline{z_{e}}}^{2}}\left(1-\frac{\overline{\mathbf{1}_{\mathrm{z}_{\mathrm{j}}}<\delta_{1} z_{e}}}{N I \bar{z}_{e}}\right)^{2}+\frac{\sigma_{I \infty}^{2}\left(\mathrm{I}_{\mathrm{b}}, \lambda_{1} \bar{\delta}\right)}{\bar{\delta}_{e}^{2}\left(1-\mathrm{I}_{\mathrm{b}}\right)^{2}}
$$

1131 With $\mathrm{I}_{\mathrm{b}}$ defined as deal with case $\mathrm{I}=1$ as in Supplementary S2:

$$
\mathrm{I}_{\mathrm{b}}=\min \left(\mathrm{I}, 1-\frac{1}{2 N+2}\right)
$$

1132 S3.6. Cramer Rao bound

1133 The Fisher information (Kay, 1993, Chapter 3) measures the amount of information that is carried about the

1134 attenuation coefficient, by the set of distances travelled by beams within a voxel $\left\{x_{i}\right\}_{i \leq N}$. It is defined as:

$$
\mathrm{I}_{F}(\lambda)=\mathrm{E}\left[\left(\frac{\mathrm{d} \log \mathcal{L}}{\mathrm{d} \lambda}\right)^{2}\right]=-\mathrm{E}\left[\frac{\mathrm{d}^{2} \log \mathcal{L}}{\mathrm{d} \lambda^{2}}\right]
$$

1135 Since the log likelihood function of Z (S3-1) is twice differentiable, the Fisher information can be expressed as:

$$
\mathrm{I}_{F}(\lambda)=\frac{\mathrm{E}\left[\mathrm{N}_{\mathrm{i}}\right]}{\lambda^{2}}=\frac{\mathrm{NI}_{\infty}(\lambda)}{\lambda^{2}}
$$


1136 The Cramer-Rao bound is the inverse of the Fisher Information:

$$
\mathrm{CRB}_{\lambda}=\frac{\lambda^{2}}{\mathrm{NI}_{\infty}(\lambda)}
$$

1137 In the case of a spherical voxel, the Cramer-Rao bound can be analytically computed (see section S4.2). 


\section{Supplementary S4. Technical derivation in a spherical voxel}

\section{S4.1. Distribution of optical depths in a spherical voxel}

1140 In this subsection, we present the derivation leading to the PDF of optical depths $\left\{\mathrm{L}_{\mathrm{j}}\right\}$ in a spherical voxel,

1141 required for the numerical simulation described in subsection 4.3 .

1142

1143 If $\mathrm{r}$ is the distance between the beam and the sphere center, the path length of the beam within the sphere is:

$$
L_{j}=\lambda \delta_{j}=\lambda 2 \sqrt{R^{2}-r^{2}}
$$

1144

1145

1146

1147

1148

1149

1150

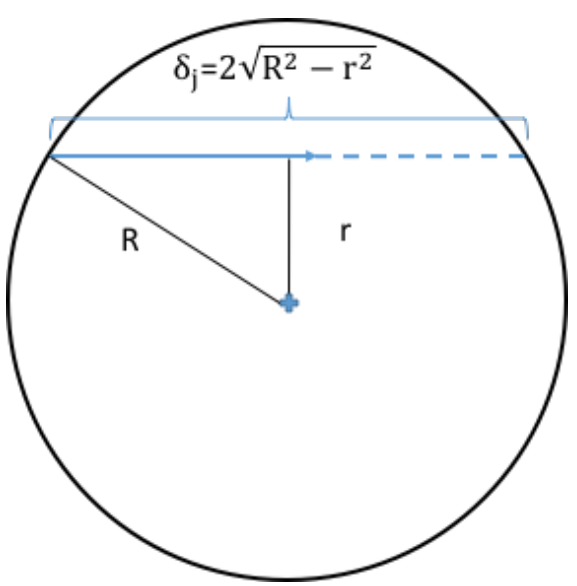

Fig. S4-1. Illustration of the numerical simulation of a TLS beam crossing a spherical voxel with radius R and path length $\delta_{\mathrm{i}}$.

Assuming a constant beam density within the sphere, the beam density with length $\mathrm{L}_{\mathrm{j}}$ is:

$$
P\left(\lambda \delta_{j}(r) \leq L_{j}<\lambda \delta_{j}(r+d r)\right)=\frac{2 \pi r d r}{\pi R^{2}}=\frac{2 r d r}{R^{2}}
$$

With $\mathrm{u}=\frac{\mathrm{r}}{\mathrm{R}}$ between 0 and 1 and $h(u)=\sqrt{1-\mathrm{u}^{2}}$, it becomes:

$$
P\left(\lambda 2 R h(u) \leq L_{j}<\lambda 2 R h(u+d u)\right)=2 u d u
$$

1151 Or equivalently, using (44):

$$
P\left(L_{j}(u) \leq L_{j}<L_{j}(u+d u)\right)=2 u d u
$$

1152 with $L_{j}(u)=\frac{3}{2} \mathrm{~L} \sqrt{1-\mathrm{u}^{2}}$

S4.2. Cramer-Rao bound for dimensionless spherical voxels

1155 As defined in Supplementary S5.2, the Cramer-Rao bound for a spherical voxel is given by: 


$$
\mathrm{CRB}_{\mathrm{L}}=\frac{\mathrm{L}^{2}}{\mathrm{NE}(\mathrm{I})}
$$

1156 For the Beer-Lambert law, the expectation of the relative density index E(I) can be expressed as a function of the

1157 optical depth Li:

$$
E[I]=1-\int_{0}^{1} e^{-L_{i}(u)} 2 u d u
$$

1158 With $\mathrm{y}=\frac{3}{2} \mathrm{~L} \sqrt{1-\mathrm{u}^{2}}, \mathrm{dy}=\frac{3}{2} \mathrm{~L} \frac{1}{\sqrt{1-\mathrm{u}^{2}}} \frac{1}{2}(-2 \mathrm{udu})$ so that $\mathrm{udu}=\frac{4}{9 \mathrm{~L}^{2}} y \mathrm{dy}$ and:

$$
\int_{0}^{1} e^{-L_{i}(r)} 2 u d u=-\frac{8}{9 L^{2}} \int_{\frac{3}{2} L}^{0} e^{-y} y d y=\frac{8}{9 L^{2}} \int_{0}^{\frac{3}{2} L} e^{-y} y d y
$$

1159 Integrating by parts:

$$
\int_{0}^{1} \mathrm{e}^{-\mathrm{L}_{\mathrm{i}}(\mathrm{r})} 2 \mathrm{udu}=\frac{8}{9 \mathrm{~L}^{2}}\left(\left[-\mathrm{ye} \mathrm{e}^{-\mathrm{y}}\right]_{0}^{\frac{3}{2} \mathrm{~L}}-\int_{0}^{\frac{3}{2} \mathrm{~L}}-\mathrm{e}^{-\mathrm{y}} \mathrm{dy}\right)=\frac{8}{9 \mathrm{~L}^{2}}\left(1-\mathrm{e}^{-\frac{3}{2} \mathrm{~L}}-\frac{3}{2} \mathrm{Le}^{-\frac{3}{2} \mathrm{~L}}\right)
$$

1160 Which leads to:

$$
E[I]=1-\frac{8}{9 L^{2}}\left(1-e^{-\frac{3}{2} L}-\frac{3}{2} L e^{-\frac{3}{2} L}\right)
$$

1161 Thus

$$
\mathrm{CRB}_{\mathrm{L}}=\frac{\mathrm{L}^{2}}{\mathrm{~N}\left(1-\frac{8}{9 \mathrm{~L}^{2}}\left(1-\mathrm{e}^{-\frac{3}{2} \mathrm{~L}}-\frac{3}{2} \mathrm{Le}-\frac{3}{2} \mathrm{~L}\right)\right)}
$$

1162

1163 


\section{Supplementary S5: Dimensionless quantities used in numerical simulations}

1165 S5.1. Finite element simulations (Described in section 4.2):

1166 The dimensionless quantities of interest for these numerical simulations are:

$$
\begin{aligned}
& \mathbf{1}_{e}=\frac{\delta_{e}}{\delta}=-\frac{\log \left(1-\lambda_{1} \delta\right)}{\lambda_{1} \delta}=-\frac{\log \left(1-\mathrm{L}_{1}\right)}{\mathrm{L}_{1}} \\
& \hat{\mathrm{l}}=\hat{\lambda} \delta= \begin{cases}-\log (1-\mathrm{I}) & \text { when } I<1 \\
+\infty & \text { when } I=1\end{cases} \\
& \hat{\mathrm{L}}=\widehat{\Lambda} \delta=\left\{\begin{array}{l}
-\frac{1}{1_{e}}\left(\log (1-\mathrm{I})+\frac{\mathrm{I}}{2 \mathrm{~N}(1-\mathrm{I})}\right) \\
\frac{1}{1_{e}} \log (2 \mathrm{~N}+2)
\end{array}\right. \\
& \sigma_{\overparen{\mathrm{L}}}^{2}=\sigma_{\widehat{\Lambda}}^{2} \delta^{2}= \begin{cases}\frac{I}{1_{e}^{2}(1-I)}\left(\frac{1}{N}+h_{\infty}\left(\mathrm{I}, \mathrm{L}_{1}\right)\right)\left(1-\frac{1}{2 N(1-I)}\right)^{2} & \text { when } I<1 \\
\frac{2 N-1}{1_{e}{ }^{2}}\left(\frac{1}{N}+h_{\infty}\left(\frac{1}{2 N+2}, \mathrm{~L}_{1}\right)\right) & \text { when } I=1\end{cases} \\
& \overline{\mathrm{y}_{e}}=\lambda \overline{\mathrm{z}_{e}}=-\lambda \overline{\frac{\log \left(1-\lambda_{1} \mathrm{z}_{\mathrm{J}}\right)}{\lambda_{1}}}=-\frac{\mathrm{L}}{\mathrm{L}_{1}} \overline{\log \left(1-\frac{\mathrm{L}_{1}}{\mathrm{~L}} \mathrm{y}_{\mathrm{J}}\right)} \\
& \overline{\mathbf{1}_{y<\mathrm{L}} \mathrm{Y}_{e}}=\lambda \overline{\mathbf{1}_{z<\delta} \mathrm{y}_{e}}=-\lambda \overline{\frac{\mathbf{1}_{z<\delta} \log \left(1-\lambda_{1} \mathrm{z}_{\mathrm{J}}\right)}{\lambda_{1}}}=-\frac{\mathrm{L}}{\mathrm{L}_{1}} \overline{\mathbf{1}_{y<\mathrm{L}} \log \left(1-\frac{\mathrm{L}_{1}}{\mathrm{~L}} \mathrm{y}_{\mathrm{J}}\right)} \\
& \tilde{\mathrm{l}}=\tilde{\lambda} \delta=\frac{\mathrm{L}}{\overline{\mathrm{y}}} \mathrm{I} \\
& \tilde{\mathrm{L}}=\tilde{\Lambda} \delta=\frac{\mathrm{L}}{\overline{\mathrm{y}_{\mathrm{e}}}}\left(\mathrm{I}-\frac{\overline{\mathbf{1}_{y<\mathrm{L}} \mathrm{y}_{e}}}{{\mathrm{~N} \overline{\mathrm{y}_{e}}}}\right) \\
& \sigma_{\widetilde{\mathrm{L}}}^{2}=\sigma_{\widetilde{\Lambda}}^{2} \delta^{2}=\frac{\mathrm{L}^{2} \mathrm{I}}{\mathrm{N}_{\bar{e}}^{2}}\left(1-\frac{\overline{\mathbf{1}_{\mathrm{y}<L} y_{e}}}{N I \overline{y_{e}}}\right)^{2}+\frac{\sigma_{I_{\infty}}^{2}\left(\mathrm{I}_{\mathrm{b}}, \mathrm{L}_{1}\right)}{1_{e}{ }^{2}\left(1-\mathrm{I}_{\mathrm{b}}\right)^{2}} \\
& \mathrm{CRB}_{\mathrm{L}}=\frac{\mathrm{L}^{2}}{\mathrm{NE}(\mathrm{I})}=\frac{\mathrm{L}^{2}}{\mathrm{~N}\left(1-\left(1-L_{1}\right)^{L / L_{1}}\right)}
\end{aligned}
$$

when $I<1$

when $I=1$

1167

\section{S5.2. Unequal path length simulations (Described in section 4.3)}

1169 The ratio of the volume to cross section of the voxel $\frac{\mathrm{v}}{\mathrm{S}}$ is $\bar{\delta}^{\infty}$, the limit of $\bar{\delta}$ when $\mathrm{N}$ tends to infinity, since a

1170 constant surface density of beams is assumed. Thus, the asymptotic optical depth is:

$$
\mathrm{L}=\lambda \frac{\mathrm{V}}{\mathrm{S}}=\lambda \bar{\delta}^{\infty}
$$

1171 With

$$
\overline{\mathrm{L}_{e}}=\lambda \overline{\delta_{e}}=\lambda \bar{\delta}
$$


1172 and

$$
\sigma_{L}^{2}=\sigma_{\delta}^{2} \lambda^{2}
$$

1173 The dimensionless quantities of interest are:

$$
\begin{aligned}
& 1_{e}=\frac{\overline{\mathrm{L}_{e}}}{\mathrm{~L}} \\
& \hat{\imath}=\hat{\lambda} \delta=\left\{\begin{array}{l}
-\frac{1}{1_{e}} \log (1-\mathrm{I}) \\
+\infty
\end{array}\right. \\
& \hat{\mathrm{L}}=\widehat{\Lambda} \delta=\left\{\begin{array}{l}
-\frac{1}{1_{e}}\left(\log (1-\mathrm{I})+\frac{\mathrm{I}}{2 \mathrm{~N}(1-\mathrm{I})}\right) \\
\frac{1}{1_{e}} \log (2 \mathrm{~N}+2)
\end{array}\right. \\
& \hat{\mathrm{L}}_{2}=\widehat{\Lambda}_{2} \frac{\mathrm{V}}{\mathrm{S}}=\frac{\mathrm{V}}{\mathrm{S}} \frac{\bar{\delta}}{\sigma_{\delta}^{2}}\left(1-\sqrt{1-2 \frac{\sigma_{\delta}^{2}}{\bar{\delta}} \widehat{\Lambda}}\right)=\frac{\mathrm{L} \overline{\mathrm{L}_{e}}}{\sigma_{\mathrm{L}}^{2}}\left(1-\sqrt{1-2 \frac{\sigma_{\mathrm{L}}^{2}}{\mathrm{~L}_{e}} \hat{\mathrm{L}}}\right) \\
& \sigma_{\widehat{\mathrm{L}}}^{2}=\sigma_{\widehat{\Lambda}}^{2}\left(\frac{\mathrm{V}}{\mathrm{S}}\right)^{2}= \begin{cases}\frac{I}{1_{e}^{2} N(1-I)}\left(1-\frac{1}{2 N(1-I)}\right)^{2} & \text { when } I<1 \\
\frac{2-\frac{1}{N}}{1_{e}{ }^{2}} & \text { when } I=1\end{cases} \\
& \sigma_{\widehat{\mathrm{L}}_{2}}^{2}=\sigma_{\widehat{\Lambda}_{2}}^{2}\left(\frac{\mathrm{V}}{\mathrm{S}}\right)^{2}=\sigma_{\widehat{\mathrm{L}}}^{2}\left(1+\frac{\sigma_{\mathrm{L}}^{2}}{\overline{\mathrm{L}}_{e}} \hat{\mathrm{L}}\right)^{2} \\
& \mathrm{CRB}_{\mathrm{L}}=\frac{\mathrm{L}^{2}}{\mathrm{~N}\left(1-\frac{8}{9 \mathrm{~L}^{2}}\left(1-\mathrm{e}^{-\frac{3}{2} \mathrm{~L}}-\frac{3}{2} \mathrm{Le}-\frac{3}{2} \mathrm{~L}\right)\right)} \\
& \text { when } I=1
\end{aligned}
$$


1175

1176

1177

1178

1179

$$
\begin{aligned}
& \overline{\mathrm{f}(\mathrm{X})} \approx \mathrm{f}(\overline{\mathrm{X}})+\frac{1}{2} \sigma_{X}^{2} f^{\prime \prime}(\bar{X}) \\
& \operatorname{Var}(\mathrm{f}(\mathrm{X})) \approx\left(\mathrm{f}^{\prime}(\overline{\mathrm{X}})\right)^{2} \sigma_{X}^{2} \\
& \overline{\mathrm{f}(\mathrm{X}, \mathrm{Y})} \approx \mathrm{f}(\overline{\mathrm{X}}, \overline{\mathrm{Y}})+\frac{1}{2} \sigma_{X}^{2} \frac{\partial^{2} f}{\partial x^{2}}(\overline{\mathrm{X}}, \overline{\mathrm{Y}})+\frac{1}{2} \sigma_{Y}^{2} \frac{\partial^{2} f}{\partial y^{2}}(\overline{\mathrm{X}}, \overline{\mathrm{Y}})+\sigma_{X, Y} \frac{\partial^{2} f}{\partial x \partial y}(\overline{\mathrm{X}}, \overline{\mathrm{Y}}) \\
& \operatorname{Var}(\mathrm{f}(\mathrm{X}, \mathrm{Y})) \approx\left(\frac{\partial f}{\partial x}(\overline{\mathrm{X}}, \overline{\mathrm{Y}})\right)^{2} \sigma_{X}^{2}+\left(\frac{\partial f}{\partial y}(\overline{\mathrm{X}}, \overline{\mathrm{Y}})\right)^{2} \sigma_{Y}^{2}+2 \frac{\partial f}{\partial x}(\overline{\mathrm{X}}, \overline{\mathrm{Y}}) \frac{\partial f}{\partial y}(\overline{\mathrm{X}}, \overline{\mathrm{Y}}) \sigma_{X, Y}
\end{aligned}
$$

1180

1181

(S6-1) and (S6-3) are used to compute the bias correction for the estimators of $f(\bar{X})$ and $f(\bar{X}, \bar{Y})$, since

$$
\overline{\mathrm{f}(\mathrm{X})-\frac{1}{2} \sigma_{X}^{2} f^{\prime \prime}(X)} \approx \mathrm{f}(\overline{\mathrm{X}})
$$

1182

And

$$
\overline{\mathrm{f}(\mathrm{X}, \mathrm{Y})-\frac{1}{2} \sigma_{X}^{2} \frac{\partial^{2} f}{\partial x^{2}}(\mathrm{X}, \mathrm{Y})-\frac{1}{2} \sigma_{Y}^{2} \frac{\partial^{2} f}{\partial y^{2}}(\mathrm{X}, \mathrm{Y})-\sigma_{X, Y} \frac{\partial^{2} f}{\partial x \partial y}(\mathrm{X}, \mathrm{Y})} \approx \mathrm{f}(\overline{\mathrm{X}}, \overline{\mathrm{Y}})
$$

1183

1184

Proof:

1185

1186

The proof of (S6-1) is the following. For a set of $\mathrm{N}$ value $\mathrm{x}_{j}$ of the random variable $\mathrm{X}$ :

$$
\mathrm{f}\left(\mathrm{x}_{j}\right)=\mathrm{f}\left(\overline{\mathrm{x}}_{J}+\left(\mathrm{x}_{j}-\overline{\mathrm{x}}_{J}\right)\right) \approx \mathrm{f}\left(\overline{\mathrm{x}}_{J}\right)+\left(\mathrm{x}_{j}-\overline{\mathrm{x}}_{J}\right) f^{\prime}\left(\overline{\mathrm{x}}_{J}\right)+\frac{1}{2}\left(\mathrm{x}_{j}-\overline{\mathrm{x}}_{J}\right)^{2} f^{\prime \prime}\left(\overline{\mathrm{x}}_{\mathrm{J}}\right)
$$

1187 Summing over N and dividing by $\mathrm{N}$ leads to:

$$
\frac{1}{N} \sum_{j=1}^{N} \mathrm{f}\left(\mathrm{x}_{j}\right) \approx \mathrm{f}\left(\overline{\mathrm{x}}_{J}\right)+\frac{f^{\prime}\left(\overline{\mathrm{x}}_{J}\right)}{N} \sum_{j=1}^{N}\left(\mathrm{x}_{j}-\overline{\mathrm{x}_{J}}\right)+\frac{1}{2} \frac{f^{\prime \prime}\left(\overline{\mathrm{x}_{J}}\right)}{N} \sum_{j=1}^{N}\left(\mathrm{x}_{j}-\overline{\mathrm{x}}_{J}\right)^{2}
$$

1188 The second term is by definition equal to 0 . Thus, with $\sigma_{X}^{2}$ the usual unbiased estimates for variance:

$$
\overline{f\left(x_{J}\right)} \approx \mathrm{f}\left(\overline{\mathrm{x}}_{\mathrm{J}}\right)+\frac{1}{2} \frac{N-1}{N} \sigma_{X}^{2} f^{\prime \prime}\left(\overline{\mathrm{x}}_{\mathrm{J}}\right) \approx \mathrm{f}\left(\overline{\mathrm{x}}_{\mathrm{J}}\right)+\frac{1}{2} \sigma_{X}^{2} f^{\prime \prime}\left(\overline{\mathrm{x}}_{\mathrm{J}}\right)
$$


1190 The proof of (S6-2) is similar:

$$
\left(\mathrm{f}\left(\mathrm{x}_{j}\right)-\mathrm{f}\left(\overline{\mathrm{x}}_{\mathrm{J}}\right)\right)^{2} \approx\left(\left(\mathrm{x}_{j}-\overline{\mathrm{x}}_{\mathrm{J}}\right) f^{\prime}\left(\overline{\mathrm{x}}_{\mathrm{J}}\right)\right)^{2}
$$

1191 Thus,

$$
\frac{1}{N-1} \sum_{j=1}^{N}\left(\mathrm{f}\left(\mathrm{x}_{j}\right)-\mathrm{f}\left(\overline{\mathrm{x}}_{\mathrm{J}}\right)\right)^{2} \approx \frac{f^{\prime}\left(\overline{\mathrm{x}}_{\mathrm{J}}\right)^{2}}{N-1} \sum_{j=1}^{N}\left(\mathrm{x}_{j}-\overline{\mathrm{x}}_{\mathrm{J}}\right)^{2}
$$

1192 So that,

$$
\frac{1}{N-1} \sum_{j=1}^{N}\left(\mathrm{f}\left(\mathrm{x}_{j}\right)-\mathrm{f}\left(\overline{\mathrm{x}}_{\mathrm{J}}\right)\right)^{2} \approx f^{\prime}\left(\overline{\mathrm{x}}_{\mathrm{J}}\right)^{2} \sigma_{X}^{2}
$$

1193

1194 Similar derivations for a function of two variables lead to (S6-3) and (S6-4). Then (S6-5) and (S6-6) follow. 